\title{
Archaeology of Human Consciousness: An Integrated Narrative of Cognitive Evolution from the Preanthromorphic Mind to Humanity's Contemporary, Academia-Centric Culture
}

\author{
Eric Bond \\ Whitman College, Walla Walla, Washington, USA \\ Email: enrique5043@outlook.com
}

How to cite this paper: Bond, E. (2021). Archaeology of Human Consciousness: An Integrated Narrative of Cognitive Evolution from the Preanthromorphic Mind to $\mathrm{Hu}$ manity's Contemporary, Academia-Centric Culture. Advances in Anthropology, 11, 201-248.

https://doi.org/10.4236/aa.2021.113013

Received: July 7, 2021

Accepted: August 13, 2021

Published: August 16, 2021

Copyright $\odot 2021$ by author(s) and Scientific Research Publishing Inc. This work is licensed under the Creative Commons Attribution International License (CC BY 4.0).

http://creativecommons.org/licenses/by/4.0/

\begin{abstract}
A comprehensive model of the human intellect's evolution is presented, ranging from the origins of higher cognition to 21 st century academic culture. It begins by delving into the composition of the pre-human mind, treating complications in deriving an all-encompassing theory of life's comparative phenomenology, and then providing outlines of such a theory to the extent which seems possible at our current stage of knowledge. Parameters in the evolution of communication and language are set out along with the way these factors converged to produce the linguistic properties of human thought and behavior. These insights are combined in a phenomenology of the anatomically modern human psyche, coupled with a narrative of humanity's evolution from prehistoric lifestyles to civilization of the historical period. This is followed by a description of the evolution of Western discourse away from essentialist illusions and towards a more versatile perspectivism.
\end{abstract}

\section{Keywords}

Archaeology of Consciousness, Evolutionary Phenomenology, Higher Cognition, Evolution of Language, Evolution of the Modern Human Mind, Prehistory, Cultural Evolution, Development of Western Academia

\section{The Evolution of Human Life}

In the 18th century, Swedish naturalist Carl Linnaeus wrote, "I well know what a 
splendidly great difference there is between a man and a bestia when I look at them from a point of view of morality. Man is the animal which the creator has seen fit to honor with such a magnificent mind and has condescended to adopt as his favorite and for which he has prepared a nobler life." This neatly encapsulates the transition between two key eras in history, a Europe of the Middle Ages/Early Modern period, dominated by religious thinking that spiritually exalted our species' privileged place in the planet's web of life, and the post-Enlightenment modernity increasingly centered around mechanistic, theoretical and empirical progress, responsible for proving that humans are just one intelligent species among thousands, which hit the jackpot of technological savvy at the expense of sustainability, for civilized history has been relentlessly punctuated with collapses induced by slight deviations in climate, diseases exacerbated by crowding, weaponized combat and more, altogether infused with rampant logistical misjudgment. More generally, the quote illuminates a frequently conflicted hubris of human individuals regarding their own nature: we are animals subjected to all the exigencies, discontents and perils of a ruthless ecosystem, but tend to see ourselves as existing on a higher plane than other species, an outlook is not without plausibility, for civilization is undeniably unique amongst Earth's organisms and has led to an unprecedented facility in shaping the environment. Humankind is capable of a penetrating reason while distinctively cultural, yet also remains the slave of almost compulsory drives, with all of this forged by prehistoric wilderness lifestyles and eons-old evolutionary forces comparable to those operatives upon the rest of our planet's species.

The most significant distinction between humans and other animals must largely reside in cognition's contribution to our lifestyles, for it is the way we think which allows us to perform all the feats that make the species' predominance possible. We might hone in on language ability and its impact on reasoning as the feature distinguishing us from all other species, but it has been demonstrated that dolphins vocalize at a faster rate and perhaps with more complexity than humans. Social cohesion could be our advantage, but bees, ants and many more species have brains that allow them to live in collectives rivaling humanity's organizational efficiency. The capacity to educate may be what sets us apart, but numerous species besides humans teach cohorts how to find food, construct nests, and fit in with a social group. Technological creativity obviously seems to be one of our edges, but without tens of thousands of years of cumulative learning behind us, would we be any more capable of fashioning implements than a chimpanzee?

The convergence of characteristics that make us human is undoubtedly unique, but for any biological trait we consider, something like it can be found in another organism. Since all of these species who share at least part of our profile have a common ancestor with humans, the originating seeds of much of our cognitive makeup must be located far in the past, even prior to the first mammals. And it is not so much how we behave and relatedly think that is entirely without peer, 
but rather what we think about. Raw physiological material of the organic mind was crafted by evolutionary influences for hundreds of millions of years, then slightly altered or reconfigured somehow in our lineage during the most recent two hundred thousand to build an unparalleled edifice of what humans conceive, causing civilized culture to leap out of the wilderness, revolutionizing our species' way of life and utterly remaking the globe. The goal of this paper is to explicate the transition from preanthromorphic intelligence to contemporary civilization, highlighting sequences of development which gave rise to anatomically modern humans and then the modern world. This will clarify where we came from, where we are going, and the spectacular potential for actualization that our human future holds.

\section{Humanity and the Evolutionary Phenomenology of Preanthromorphic Cognition}

The period from hominid evolution to human civilization is unprecedented in Earth's history. Fossil records reveal that the majority of our planet's past has been comprised of equilibriums lasting from tens to hundreds of millions of years, during which the composition and distribution of species remained relatively static. Primates have been around for more than 50 million years; the reign of mammals has lasted from the extinction of dinosaurs approximately 65 million years ago to present; dinosaurs and their reptile like ancestors diversified and predominated soon after the Permian extinction 250 million years ago; eukaryotic life began to assume the macroscopic features characteristic of modern ecosystems upon the Cambrian explosion roughly 550 million years ago, with contemporary forms of photosynthetic and metabolic chemistry, internal and sense organs, limbs for motility, jaws for predation, sense organs, gills, fins, wings, and fertilization of egg with sperm all entrenched for more than 400 million years (Scientific Psychic, n.d.). It has become apparent that adaptive radiation happens with rapidity once an ecosystem is destabilized by depopulation or a particularly advantageous trait develops, but until the advent of our own Homo genus, these transition periods tended to be constrained by what we acknowledge as physical environments and bodies: climate, availability of minerals, size, speed, sensory recognitions, rates of reproduction, or survival-related physiology and behavior (Berkeley.edu, n.d.). Descent is clearly demarcated within stratifications of sedimentary rock as eras lasting tens of millions of years, and while there are plenty of fascinating surprises, we have no trouble imagining what the whole of these ancient worlds looked like in comparison to wildernesses of our own time. Organisms with much resemblance to those we see today have been growing, swimming, crawling, burrowing, flying, scurrying, scampering, intruding into each other's metabolic and reproductive business in line with principles of nonhuman population dynamics, food chains and nutrient cycling for 2000 times as long as Homo sapiens' existence, and 100 times as long as the ancestral bipedalism anything like what we would distinguish as closer to a human than to a bonobo or chimpanzee (Tropical Essays, 2011). 
This provides stark contrast to the evolution of Homo sapiens from the first hominids such as Austrolepithecus. In around four million years, a walking anthropoid species with what was in all probability chimplike intelligence went from subsisting sparsely in Africa to diversifying into multiple Homo species, most prominently Homo habilis, then Homo erectus, Homo heidelbergensis and Homo neanderthalensis, whose ranges spanned the entirety of the Old World by two hundred thousand years ago (PBS, n.d.). These species engaged in technological behaviors that were novel for Earth's organisms, fabricating tools for hunting, gathering and storing (Welker, n.d.). This is intriguing, but pales in comparison to the consequences of our own species' origins: in two hundred thousand years, anatomically modern humans occupied every land mass on the planet, successfully adapting to all but the harshest environments on Earth, either absorbing or displacing the rest of the genus. We displayed problem-solving and aesthetic sensibility of a high functioning nature, disjuncted from anything that had yet arisen: archaeological finds dated to more than a hundred thousand years ago reveal the first signs of deeply symbolic art and artifacts, what would become the aforementioned global wanderlust, and surplus capacity to arrive at solutions for dilemmas posed by natural environments, eventually including prehistory's first ecological methods such as deforesting with fire and cultivating plants (Harcourt, 2015).

Cosmopolitanism of the human race was abetted during the most recent ice age, which peaked roughly twenty thousand years ago. Sea level dropped a hundred feet, connecting formerly inaccessible regions to mainland Asia, most notably the Western Hemisphere by way of a land bridge between what are modern day Siberia and Alaska (Science.smith.edu, n.d.). As world climate became more hospitable from twenty to ten thousand years ago, human populations throughout the world swelled, and proto-civilized mingling between hunter-gatherers seems to have grown commonplace, with remnants of expansive meeting grounds dated to this period found in many locations (Torrey, 2017). No later than ten thousand years ago, humans had achieved enough proficiency in the selective breeding of plants and animals to enable a lifestyle centered around farming, probably driven by trading of ideas and techniques as well as the necessity that larger, more concentrated food supplies be secured for bigger populations (History.com., n.d.). Transition to civilized living took place in fits and starts, cycling between dispersals and reorganizations over thousands of years as fluctuations in climate repeatedly rendered rudimentary methods of agriculture inadequate. But by the 6th or 5th millennium B.C.E., food production had reached an advanced enough stage in some regions, such as Mesopotamia, Egypt, China and soon Europe, that civilization was able to lay down permanent roots (History.com., n.d.). Economies, political institutions and technological development got up and running, along with the first written records, then artistic and philosophical literature, ultimately seeding a cumulative discourse attempting to understand nature and the cosmos in terms of its seemingly systematic principles, which gave rise to academics and methodological empiricism in our historical pe- 
riod persisting to the present day (Schmandt-Besserat, 2014; Mark, 2009).

We have some general notions of how the genus made its way from the first hominids towards human civilization: changes in physique allowed the brain to grow larger and utilize greater amounts of the body's metabolism, technological facility steadily increased within this timeframe, full-fledged humanity is more artistic and communal than its ancestral species, and we have obviously inclined towards more intellectual refinement than other biological forms of sociality. But evaluating the causality of this transformation along with its cultural import is a vexing theoretical difficulty.

To start with, as previously indicated, macroevolution during pre Homo epochs happened via natural selection over the course of at least tens of millions of years, depositing huge amounts of evidence as to what transpired, clearly differentiated as fossilization in rock-hard groundmass, while the switch from chimp like intelligence to enculturate human cognition in technology-based civilization, unprecedented for Earth's history, materialized in only four million years, with the better part of this process ascribable to the most recent one or two hundred thousand. Combined with effacement of geologically shallow evidence by prescientific humans, we have much less to go on in conjecturing about ourselves.

Not only this, but as mentioned, earlier instances of adaptive metamorphosis and radiation differ in that they promptly reached stases sustained by material factors exacted upon macroscopic eukaryotes for at least 500 million years, instating the exigencies of nutritional acquisition, reproduction, and survival in general with all their instantiations in matter. Evolutionary success or failure of every species outside the Homo genus can be attributed primarily to bodily form and the physical purport of behavior. Our own genus differs because its cognition evolved enough sophistication that these organisms strategically remodeled environments, social arrangements and even their own selves as motivated by thoughtful, outstandingly creative intentions. Concept-formation and psychical comportment were largely liberated from restraints imposed by foregoing intrinsicalities of nature, with a brain and behavioral repertoire evolving in ways highly independent of material conditions, via more mind-centric effects on selection pressures and trait profiles, attaining the potency to change nature at a speedy pace. The first 500 million years of eukaryotic subsistence are intuitive within the bounds of a thoroughgoing paradigm for modeling life's evolution, but at current rates the next one thousand may very well be unlike anything that has ever been imagined let alone proven.

More concept-based lifestyles of our genus, proceeding towards civilized society, have left some residual signs in the physical world, again size and shape of the skull relative to body mass, throats variously conducive to language, or fabricated objects indicating in trace ways the degree of memetic complexity, but our apperception-impelled evolution is almost unembodied historically. Positioned between the primarily corporeal history of Earth prior to six million years ago and a detailed conceptual history of civilized humanity as recorded in writing 
that began roughly three thousand years ago, there is a gap that would, as the situation stands, only be filled with exacting certainty by what at first glance seems to be an impossible archaeology of consciousness.

The mind itself has left not a shred of evidence, but there are alternate approaches in constructing an account of humanity's cognitive evolution. Extensive observation of the natural world is possible, with behaviors of a hundred thousand relatively high functioning species being especially illuminating. These organisms differ amongst themselves in a vast quantity of ways, but it is clear from scrutiny that they all understand and predict each other's activity with much accuracy. Nonhuman animals experience motivations to avoid and seek out stimuli, garner a meal or mate in much the same way as we do, though our psyches seem to be sublimated into a more intricate sense of identity, purpose, communal meaning, which simply shows up as the transparency of most animal intention compared to humans we associate with. Animals sometimes appear to show affection while more in pursuit of physical sensations and satiations, but it is also the case that core cognitive structures of pain, pleasure and empathy-amygdalic and hippocampic midbrain regions, dopamine like neurotransmitters and glutamate, mirror neurons-are present in nonhuman species as well, so the biology of human to human communality is very much like that of the human to nonhuman and nonhuman to nonhuman varieties. We are not delusional if we believe pets for instance think and feel in similar ways, for it is obvious that they do; shared conditions and common interests between all kinds of species soon produce mutualized socialities witnessed all around us. At this stage of scientific knowledge, it is premature to attempt a comprehensive elaboration of comparative psychology by referencing either intention or perceptual and behavioral physiologies, but we can nonetheless fashion a picture of cognition which proximate the truth in its essentials. The foundations of our own cognizing seem biological and considerably trans-species, provisional of an introspective naturalism.

Infused into this broad layer of cognitive function within nature is the Homo genus' evolutionary contribution to the human psyche. Direct observation is of course unrealizable at this time unless we pull off some unlikely mad scientist feat such as cloning a hominin or hominid, but even so we have some information to go on, particularly if we restrict our examination to the domain of reasoning as opposed to psychology in general. The range of moods, fantasies, delusions, unconscious wildness to which the human race is subjected defies cohesive classification at the current stage of science and philosophy, though perhaps this knowledge is within reach of techniques such as psychoanalysis, but when our minds are brought into adjacency with basic fact in thinking about the practical ramifications of cause and effect during acts of technical problem-solving, it seems most pull themselves together at work or in public, so that a functional sphere, relatively uniform cognitively, stands out in the behavioral foreground, which performs according to more universal and thus generalizable 
principles. When human beings grapple in largish collectives with concerns held in common, during which time explicit, maximally justified and comprehensible decisions must be made, these individuals coalesce into a formal sort of cognizing distinguished by the logic like standards it employs to assess meaning. This mode of thinking can be called "civic", and is the foundation for institutional operations, so that the conditions of the possibility of civically minded cognition are the conditions of the possibility for civilization's organizing. Thus, if we formulate a model of the mental modules involved in civic-focused thinking, we have made significant progress towards defining the psychology of civilized culture in contrast to every other form of sociality, and adumbrated much of what has to be explained in order to theorize conversion from hominid existence to the historical period.

So we have an array of puzzle pieces that are prerequisites of human cognition in general. Some of these are so pervasive in nature that they stand out as a near universal transhumanality, though with innumerable variations upon the main themes, and some are salient as universalities of historical civilization, responsible for its very possibility. We toss many pieces of this puzzle that we currently possess into a single pile, and anticipate connecting two broad forms in the complete image: a model of the intelligent mind as such and a model of the human mind as suited for civic reasoning. The generalities of intelligence are indispensable for human reasoning, and the faculties of formalized, institutional reasoning make the difference between mere intelligence and indispensabilities of civilized thought. The partial image etched into some puzzle pieces will be easy to identify and put in its proper place, while others could be more obscure and uncertain. As we put the pieces in order, we may find that we lack some of them, but those that are absent might be suggested with enough precision by surrounding segments to construct working hypotheses postulating their nature. So let's start fiddling with the puzzle pieces and see if a combination of fact and intuition can lend an understanding of how details constituting the entire image fit together.

Experience derives from a basic qualitative contexture consisting of core patterns in phenomenal content, also auxiliary sensations of the outside world which increase the efficiency of recognition and response to some of the most prevalent aspects of Earth's environments such as electromagnetic radiation, sound waves and chemical trails, as well as representational memory priming organisms for reaction to stimuli via sensitization and habituation. The many particulars and modules of these qualitative manifolds vary considerably between organisms, and neuroscientific psychology has not established with certainty what the substance of firsthand experience is or even how it adheres to the physique, though resonant combinations of quantum entanglement and additive superposition amongst matter are a plausible hypothesis (Bond, 2020b), but if one looks to pinpoint as inclusively as possible the essence of what makes perception functional in the context of organic life, it is probably synchronization. 
Though the difference in qualitative modularity between organisms is vast, also with much moment to moment variation even in single individuals, this activity becomes a mind by virtue of its coordination, some binding property uniting disparate cognitive components, a dynamic of integration for carrying out perceptual interpretations which syncs the phenomenal with differentiated and often far-flung physiology. We can preliminarily surmise that this synthetical agent is at least in part the electromagnetic field supervenient on an organism's body, with especially strong presence in the nervous system and brain, alongside some kinds of CPU like clock mechanism embodied in tissue (Bond, 2020b; Mc-Fadden, 2021; Bond, 2020c).

As a subcomponent of qualitative contexture, the mind evolved a faculty for interfacing phenomenal experience with both environment and body on larger scales, the association-making substratum as well as evolutionary precursor of humanesque minds, which seems to exist in most macroscopic species, from the lowliest worms, to kinds of insects that can live without their heads for days, to moderately cognitive species such as most birds and mammals, to the most high functioning like great apes, dolphins or humans. This consists in functionality orienting the organism within a perceived positional matrix, similarly instantiated in a vast quantity of species, from the extremely simple to the most complex and conceptual, together with inclinations of attraction and repulsion that are more malleable and which can be extremely species specific.

The existence of a positionalizing awareness, which 18th century philosopher Immanuel Kant and more paint with rather broad strokes as space and time, is obvious enough that examples probably do not have to be provided, for most species are clearly sensing a general location in relationship to surroundings, instinctively navigating the substrate of objects, forces, textures of aggregate matter we all reside in, a capacity which appears to be almost completely hardwired. The physiological scale at which this orientational sensibility takes effect is excellent fodder for biological research; science can determine whether it is linked to nervous tissue in some way, cellular biochemistry in general, or otherwise.

Attraction and repulsion in organisms is dazzlingly varied. Ants are drawn to pheremonal signatures. Honey bees have pheremonal sensitivity also, love to slurp nectar, and seem to get off on the bright colors and intricate shapes of flowers. The smell of kitty litter prompts cats to seek out and do their business in litter boxes. Protozoa dart away from sudden brightness and back into the dark. Insects are averse to large vibrations and for good reason, usually engaging in some kind of evasion maneuver such as fleeing or attempting to camouflage themselves with motionlessness. These tendencies to seek out or withdraw from stimuli as initiated by sensing, anatomy and biochemistry, along with co-occurring phenomenal perceptions, are naturally selected in conjunction with the mutating relationship between body and environment. Bee awareness keys in on pheremones, certain small objects, the colors and shapes of other bees, insects and flowers, or vibrations and additional signatures produced by large organisms when they 
may pose a threat, a narrowed selection of substance attributes most relevant to the functions of their bodies as exacted by mouthparts, stingers, wings, compound eyes, nesting and swarming. Humans are attracted to symmetry, musical sound as composed of numerical ratios between changing pitches, or the appearance of genitalia, and repulsed or bothered by sulfuric smells, sudden loud noises, and often the mere thought of their own past or possible pain. Any explanation of humanity's perception is of course more complex than in the case of bees, for a permeative conceptualizing renders our mental activity and resultant behavior more variegated, with most well-educated human cognition able to subtly modulate almost all instinct via intentionality. Nevertheless, we have similarly been conditioned by physiological and evolutionary pasts, factors which are intimately tied to the nature of our bodies.

How did the type of cognitive activity we might term "conception" emerge? Demystifying the processes of evolutionary development that gave rise to it is uncharted territory, but its apparent locus in modules of the brain clearly indicates that it must have appeared as a sequence of accretions augmenting some limitations in the interfacing functions of positional matrixing, attraction and repulsion previously described, allowing for greater degrees of specialized association-making as well as structures providing executive control in order to sync the disparate facets of expanding minds. Some of this enhanced association-making is what we call "thought", and the executive meta-organization of proliferating modularity in cognition is what we refer to as "self".

Thinking, a further associational integrating of perception, seems firmly attached to the nervous system and principally brain matter. Cognitive configuring involved enhances the mind's representation of environments, increasing the quantity and duration of phenomenal and physiological particulars that stimulus/response can confect and coordinate amongst at a given time, essentially diversifying and prolonging memory and its utilization in conjunction with extremely rewritable, neuromaterial types of tissue (Hampton, 2019).

So the basic qualitative contexture with its form-giving and aesthetic perceptualizations is supplemented by higher order functions that participate in differentiating and arranging it, conceptualizations which are the rudiment of ordered imagination or "reasoning". This makes minds more adaptable to changing conditions in the body and environment, hybridizing experiences which are farther removed from each other so as to better orchestrate preexisting phenomenalilty, drive and impulse, as well as platforming new kinds of qualitativity such as math, language, and various kinds of causality. The defining feature of this conceptualizing domain, to the limited extent that it can be segregated from the perceptual substratum, is probably compound contextualization, for its role is to observe fluctuating percepts constituting the kaleidoscope of mind in a sort of paralleling suspension of libido discharge, which picks out obscurer patterns by internalized motivity and fixes them into composite cognitive forms. The most accessible example of this is simply our human technical sense, how the body 
and its milieu of objects manifest to our minds as sharply relieved relations between multitudes of causes and possible effects, in most cases including entirely potential motions and purely conceptual meanings, a synthetic framework parsed by symbolic and abstract boundaries that are much greater in implication than the immediate phenomena themselves.

Along with this conceptual association-making called "thought", the mind evolved a means for exerting control over which of its brain regions and other nervous system components are active at a given time, what is approximately referred to as the "self". This is the source of basic intentionality, found throughout the animal kingdom. While lacking command of most mental activities, such as vision, hearing, startling or noticing, which are all mostly unconscious, we can rapidly bring collections of these unconscious factors into synchronous alignments at will, a sort of mode-selection phenomenon generating overall dispositions via executive mechanism. These amalgamating states of intentionality are not freely chosen to the point of independence from context, for they get sculpted over time with conditioning as well as directed by instantaneous cues from both environments and the unconscious mind itself, but we can readily carry out feats such as waking ourselves up, suppressing affect in order to focus while we reason, purposely blocking out external stimuli, as well as adopting various social and communicative strategies.

Whether association-making thought or a mode-selecting self were the originating feature that initialized evolution of the conscious mind is a bit of a chicken and egg problem; which came first? It is not clear at this stage of science if the question can even be answered, but defining "self" in terms of its anthromorphic form, as a phenomenon of introspective reflection, leads us to suspect that at least from this perspective, associational thinking was egg to the selfs chicken, an incredibly ancient type of cognitive modulization which preceded humanlike self-awareness and contributed to its construction. Regardless, it is clear that the interaction of thinking with self tended towards synergy in many lineages over vast spans of time, hundreds of millions of years, built up into more elaborate forms of pattern processing and intentionality, a richer conceiving that in essence amounts to a distinctive "presence of mind" (Bond, 2020c).

The main mutative innovation in the realm of intention was an ability to concentrate, sustaining attentive states for longer timespans, allowing keener observation of both environments as well as the organism's own phenomenal mind, a selection mechanism for associational thinking to become more astute. Thought simultaneously evolved towards greater apprehension of order amongst patterns until protological awareness had developed, an intuitive knack for grasping some prevalent kinds of cause and effect, fitting phenomenal interactivities into a kind of conceptualizing chassis of which the simplest qualities are those enumerated as basics of formal logic: negation (not p), conjunction (both $\mathrm{p}$ and $\mathrm{q}$ ), disjunction (either p or q), conditional (if p then q), and similar notions (Nolt, 2012). Association-making aptitude as logic's precursor, together with better focus, ca- 
pacitated problem-solving creativity that is a hallmark of species with the most elite technical thinking, a suite of traits we single out as elementary intelligence.

While mode-selection mechanisms we experience as the self progressed, some species gained greater cognitive control over affect, a function of suppression, sublimation, and in some cases, such as with humans, the repressing of drives and impulses by means of a subordinated unconscious. Behavior became less likely to exhibit as exhaustive discharge of libido, overpowering to intentionality, but instead compulsion and willing fused into conscious/unconscious complexes, a more cohesive and minutely modulated purposefulness, and the origins of psychical composure, which seems adaptive for the maintenance of durable social arrangements extended beyond immediate kin in more cerebral species. Some examples are cooperative hunting in wolf packs, orca pods and prides of lions, where individual initiative is sublimated into the joint venture of ritualistically seeking prey, while cathartic release is delayed or resisted altogether in order to pursue the collective goal, and selfishness largely submerged below thresholds of conscious decision-making. Grooming in primates fills a similar role, sublimating affect into gestures of amicable intention. With the self in greater charge, mobilized to regulate and protract balanced mental states, reasoning is more deliberate and socializing reflection-based so that communal identities take shape, deeper personalities partaking in a sort of protocultural awareness (Smith, 2002).

At this point, a summary of top-tier cognition might be in order. It is founded on qualitative contextural forms existing variably in very many and perhaps all species, embedded with a perceptual substratum of positional awareness along with more species-specific aesthetic penchant, added upon by specialized association-making processes and their synesthesias, which are largely responsible for the richer interpretations of causality we know as thought. A mode-selecting function exerts some executive control over which collections of cognitive modularity activate at a given time, what we experience as the intentional self. Thought and intention coevolved in many populations such that protological intuitions of associational structure and heightened attention span for applying these improved notionalizings blossomed into an intelligent mind with acumen in technical problem-solving. Intelligent intentionality often evolves greater self-control arising from an integration of reasoning with affect, restraining and diverting instinctual drives with their attendant cravings and stresses, in many cases sublimating into group standards, disciplined individuality, and mutually conceptualized identities, adaptive for tighter knit communality, bringing about conversion from simple crowding, territorializing and kin bonding to protocultural kinds of collectivity.

Well-developed cognition of a protocultural type is not what secernates humanity from the rest of nature, for many birds and mammals display it, species with intellects which must have been vestigializing and collectivizing in sociality since the origin of their common ancestor eons before extinction of the dino- 
saurs. Organisms everywhere show the signs of finespun qualitative experience, including positional sense, aesthetic preferences, thought and intentionality, with ecosystems throughout the world inhabited by intelligent selves retaining a strong, conceptually driven solidarity. Mental lives of Homo sapiens have much in common with the rest of the animal kingdom, but there are of course some obvious distinctions, chiefly language, technology and civilization. Humans are constantly emitting a stream of convoluted verbiage in large-scale, highly organized institutional settings while utilizing a fulgurous range of tools and devices, singular for planet Earth. Let's spend some time examining this uniqueness, where it started and how we arrived at our current, civilized phase of acculturated existence, as well as what it means epistemologically.

\section{Phylogenetic Factors and Evolutionary Origins of Humanity's Language and Conception}

The essence of communication in nature is discrimination of patterns produced by organic structures, allowing these structures to identify and respond to each other. Biochemical pathways within organisms perform their reactions when molecular stimulus is of a type and within a triggering threshold such that it functions as an appropriate signal, the basis for processes of interaction within and between cells, modulated by intricate systems of feedback mechanism. In lifeforms with nervous systems and accompanying perception, communicative signaling relies on recognitions of stimulating interaction by sense organs and neuromaterial tissues, chain reactions both towards and from out of the organism that meet as externalized discharge of bodily behavior, involving substantial levels of conception in the most neurally advanced species such as birds and mammals.

Perceptuality is built around the ability to hone in on aspects of substance as phenomenal attributes, which orients the organism to causality such that it can navigate the world effectively, finding food, reproducing, and doing whatever else is necessary for survival, or in the case of humans, seeking psychologically deeper actualizations as well. In many species, perceptual capacities are relatively hardwired, resulting in reflexive stimulation, but to the extent that an organism thinks in a humanlike way, it associationally links attributes independent of bodily stimulus, solely within the mind. Intentionality exerts its association-making capacity to fashion cognitive schemas, figuring out the character of attributes without having to necessarily act on its realizations by way of the physique's fumbling and prodding of environments. Absent a well-developed mind, physiological comportments are tailored, as a hybrid modularity, for targeting and picking out modularities amongst Earth's aggregate mass using increments of physical behavior, with the body only slightly more integrated by conditioning mechanisms than circumstances it is in congress with, being largely one more set of object processes. But cogitation and especially its facet of conception encompass multitudinous percepts within more unified structuralizations, a sort of mentally gen- 
erated mapping of body, phenolmenality and memory, preempting future experiences such that all of their elements are accurately allotted a proper place prior to discharge of related physical acts. This shows up intuitively in the difference between common behaviors of an individual ant and a large mammal: the ant cannot potently envisage its environment as a conceptual whole, and so when isolated must spend the majority of its time haltingly exploring its surroundings, while a puma for instance, while probably a more asocial animal in general, can envision attributes of causality as highly compound arrangements, making even its uncertainty less hesitant and more efficient. An ant pokes around in near-random fashion when on unfamiliar ground, constantly retracing its paths to eventual good fortune, but the puma decisively knows what it wants to do in more predictive manner, with greater behavioral economy.

In robustly cognitive species, communication happens as an extension of association-making intentionality, with percept attributes and the accompanying concept-tualizings becoming signals of purpose, representing symbolic meanings of and to interacting minds. For these organisms, elevation of attributes to the status of meaning springs in part from simple awareness of position, the cuing mingled with spatial and temporal sorts of separations between entities such as objects or predator and prey, which require various degrees of mental calculation to negotiate, a facet of consciousness well-represented in vertebrates, and seemingly within most more neuromaterially primitive phylums as well. There is often also a sense for territorial demarcation, the possession of one's domain in which certain behaviors by other organisms signals violation or mating interest. At this level of social consciousness, meaning is shared without yet having ascended to humanlike communality. The more cognitively advanced animals such as birds and mammals also perceive each other's bodies and actions as supplementary signs of intention, via postures, gestures, expressions or object emplacements, and these divulging corporealities are a key component of their interactions. Utterance in particular holds a vital place in projecting intent and the qualities of many experiences, from monkeys engaging in a particular howl at the sight of a predator, to dogs defending their territory with barking, to chimpanzees that compulsively emit vocalizations as they interrelate, to the syntactically complex languages of songbirds and humans. Sound production as a signaling tool, while effective for transmitting any kind of meaning, does not of course require conception anywhere near the level of human beings to be effective, and crops up in many different ways, which are however divisible into three general categories of sub-function.

Vocal behavior is driven by the impulse to discharge physiological states of arousal. Chimpanzees provide an example, as the brain regions that activate while they utter sounds are located deep in the limbic system, stimulated more by visceral affect than their creative thoughts (Cloud, 2015). This is probably typical for species without syntax, as they lack synesthesia that would synchronize numerous brain regions, such as those tied to coordination of the mouth, 
throat and bodily gesturing as well as loci of higher concepts, all of which must be integrated in order to make the most symbolic and abstract kinds of expression possible, though wherewithal to discern causality as an individual via the associating of attributes may nevertheless be quite developed.

Sound is of course one of the main mechanisms for eliciting responses from other organisms, a means by which species indicate their readiness to mate, as in a frog's croaking, or warn each other, for instance a prairie dog's territorial bark, as well as ward off potential threats, such as a snake's hiss or a bee's buzzing. To the extent that these noises signal intentions, they take on a symbolic character normalizing interactions by providing information about either the natural surroundings, for example a bee's wiggle in the hive, or cognitive disposition, in the case of a wolf's growl, a male gorilla's beating of its chest, or a deer's stomping of its front hooves. In dolphins, personal intention is sophisticated enough that their chatter includes proper names (Whitaker, 2018), and we all know how humans at the very least participate in elaborate expressiveness consisting of detailed factual content and intricate status significance.

Audibility is also useful for orienting an organism to itself and its environment. Echolocation in bats and dolphins is an example, how they issue sound to assist in steering around objects and finding food while on the move. In humans, verbalization seems to enhance cognition, as phenomenal experiences, memories and inferencing often become organized by speech or writing in ways that would not have occurred to an individual without acts of projection, something which happens for many upon merely hearing their own voice say a particular train of thought, in conversation or even only to themselves.

Sociality exacts strong selection pressure on the evolutionary development of sound production, for relationships are one of the main venues where increased complexity proves beneficial, as a supplement to coordinating mental and physical behaviors, also in displaying cognitive prowess, demonstrating intentions via audible posturing, and self-expressing. As already referenced, we easily intuit that mouths are the most readily available bodily structure by which sound transmittal can be conduced, and vocalization tends to assume a primary role in communicative behavior.

Even though these social functions of language like activity are quite uniform throughout nature, there is much variability in their particular instantiation. Humpback whale songs have been known to last for half an hour, comprised of complex, repeating themes (Editors of Encyclopedia Britannica, n.d.). Elephants communicate with subsonic vocalizations that are not audible to humans and which can travel for kilometers (Elephant Voices, n.d.). Birdsong has a complex syntax, and many of the more cognitive bird species can improvisationally mimic sounds made by other organisms (Berwick, Okanoya, Beckers, \& Bolhuis, 2011; Mayntz, 2019). Dolphins seem to be quick-witted linguistically, employing a rapid fire chatter (Whitaker, 2018). Chimps are one of the more intelligent species in terms of problem-solving adroitness, but as already mentioned, their vocalizing does 
not appear to be sophisticatedly conceptual (Cloud, 2015). And lions are highly social animals that do not seem to engage in anything approaching humanlike language, though many of their sounds do have rudimentary meanings (Kandalintseva, 2012).

Despite the relatively advanced cognition in many species, along with conceptual and emotional lives compatible enough that interspecies bonds are often possible, vocal style varies widely in line with some morphological dynamics. Larynx like structures have to be suited for the environmental medium through which sound will flow, typically either water or air, structures of hearing must be tailored to the kinds of sounds characteristic of an organism's environment and social partners, brains must be able to interpret these sounds conceptually, while species' bodies and minds of course adapt for effectively interacting with the objects and phenomena their utterances are about.

All of these animals assign meaning to sound, often in socially refined ways, and many even have some sort of syntax, so what makes our species different? Basic elements of human language are found to some degree in a large quantity of species, but our combination is a fairly unique convergence of multiple factors: huge vocabularies, as in tens of thousands of words for the average speaker; a complex and flexible syntax which can be molded into innumerable kinds of stylistic form, with much variability along the continuum of terseness or verbosity; concoction of long, inventive chains of inference, seemingly saying more with a fluid orderliness than most species; and the skill to imaginatively construct, reconstruct and deconstruct contexts in a way bolstered by linguistic communication, assimilating mountains of language-encoded information as motivated by a lifetime of hypothetical reasoning in pursuit of confirmation, refutation and integration (Lumen: Boundless Psychology, n.d.).

Even though language is a core feature of human mentality and behavior, it is not easy to comprehend. Linguistic structure is extremely synthetic, with many intricately interorganized parts - subjects, predicates, objects, syntactically connective words, tense, various shades of formality and informality - which lose a holistic impact that is difficult to introspect when not applied in what we regard as the correct manner. Yet these subunits are flexible enough that they can be applied to adequately impart meaning in such a huge host of ways that the full range of possibility tenaciously persists in defying categorization. We can say "she" to convey the concept "he", "big" can mean "small", a "hawk" is sometimes a human, syntax can be impeccably proper or flaunt conventions drastically yet stage meaning equally well, and the nature of expression completely transforms by context. Human language's vast range of meaningful formulations confounds the effort to comprehensively model its usage, and while this knowledge is probably not in principle out of reach, current theory seems insufficient for tracing modern verbalization in all its technicalities and ambiguities to the selection pressures, mutations and cognitions out of which it began. Nonetheless, interesting insights of a general kind are accessible from analyzing no more than natural se- 
lection and physiology combined with some basic characteristics of linguistic meaning.

The primary prerequisite for language's emergence is evolution into communities that are based around conceptualization, as mentioned consisting in organisms recognizing each other as well as assigning meanings to both intentional behaviors and signs of intention, so that symbols of disembodied, purely cognitive purpose are introduced to communicative interactions. The main evolutionary benefit of conceptual socializing is improved capacity to aptly and swiftly predict behavior, a ubiquitous feature of relationships between organisms with substantive nervous systems, found in probably every bird and mammal species at the least. Of course expectations can be infracted advantageously via the element of surprise, in turn prompting retributive sorts of actions, vengeance and deterrence, so a dynamic interplay of symbolic conceptualizing, anticipation and exploitation resembling human society pervades nature; however, the quantity of species with complex language is few, while human speech and writing are almost unparalleled and certainly unique. Why is human-level language ability so rare when concept-based meaning and related communications, adjudicated by what can be regarded as basic social mores, are so widespread? If we want to figure out language's presence or absence in the communities of highly cognitive species, we must outline factors distinguishing the most linguistic species from those that are less so or nonlinguistic along the communicative spectrum. To begin with, structural parameters of concept-based community should be enumerated, for this is the raw material from which linguistic behaviors are evolutionarily formed.

One main requirement is that a species' members attain baseline amounts of idle time so recreational thinking is possible, a conceptualizing free association which provides the mechanism for advances in intentional cognition, serving to reorganize brain structure and increase synesthesia in ways specifically directed by and augmentational to the self. At the most basic level, this requires that a sufficient proportion of caloric content be allocated to the brain versus the rest of the body, a criterion most species meet. There are exceptions: brachiosaurus, with that comparatively small head perched atop a massive frame, was never going to be an extraordinarily intelligent or linguistic animal, though perfectly well-suited for its milieu (Extinct Animals, n.d.). This bulky dinosaur had an additional handicap in that it probably lumbered about most of the time, grazing all day to sustain a healthy weight under the onerous demands of gravity while impervious to predators, privationed of the selection pressures and physique necessary for fast neural kinetics, in essence cognitive/behavioral quickness, a collection of traits which can be rearranged for the rapid thinking necessary in fluently inferential types of symbolic expression when leisure has reached a surfeit level. Small songbirds such as canaries by contrast, though probably no more fundamentally intelligent than a brachiosaurus and perhaps less so, move about in an ultraquick manner that is highly selective for optimizing the speed of their 
hybridized perception and mobility. When it became possible one fine day for small feathered dinosaurs to sit invulnerably for long periods of time on a tree branch, the intentional self gained more influence, expressing its modicum of libido, and speedy cognitive reflexes together with many additional vectors of formation such as mouth and throat dexterity gradually reconfigured the minds of a select few bird species for intricate, variegated and spitfire vocalization of their concepts.

In order for organisms to enter into concept-based community with potential for language, sociality must also simply be desired. Reproduction often motivates social behavior, as young of numerous species are more secure when the adults rear them in largish groups. Many birds roost collectively for the sake of progeny, the most dramatic example being Antarctic penguins that would be incapable of seeding the next generation in such a harsh climate without strategically sharing parental duties so as to keep eggs warm (Australian Antarctic Program, n.d.). Survival of adults is frequently facilitated by congregating as well, for as everyone knows, schooling, flocking and herding make prey animals less vulnerable to predators and more likely to find their way while on the move. Penguins of course must conserve energy in a low calorie environment, so incentive to expend effort on vocalization and surplus thinking is minimal, but when material factors support recreational time, more potent, in some cases conceptually linguistic cognition can result. Advanced social behavior additionally requires that an animal not be overly territorial, for sharp boundaries drawn between individuals instate less selection pressure for communication, with access to resources restricted such that procuring food is more time-consuming, an effect especially noticeable in nonlinguistic species such as pumas or tigers that have relatively high levels of intelligence but spend much of their time hunting alone. And finally, an organism's mental states, particularly its affect, must compel it to want a socialized environment. This probably makes the difference between birds, which spend much of their time in sizable flocks, and many predatory mammals that can be found operating alone or in groups of only a few individuals. The mental disposition that draws animals to gather into large, permanent arrangements can be missing, and language like vocalization materializes only under conditions of extensively close contact between many familiars, but even then very rarely.

Audible syntax is so uncommon despite the fact that these linguistic species' circumstances, conceptual faculties and communality can have much resemblance to thousands of others because it calls for massive amounts of finely tuned synesthesia. Brain regions responsible for thinking, perception of the environment, social perception, affect, as well as movement of the throat, mouth, tongue and diaphragm must all work together, dozens of specialized cognitive centers. Chimpanzees, though one of the most intelligent species overall, can do no more than separately memorize some hundred symbols and express most of these by gesturing rather than with articulated sounds, for their conceptualizing 
is neurally unconnected to the musculature and affective states of vocalization (Cloud, 2015). Parrots are intelligent birds that mimic human phrases, but lack much sense for syntax (My Animals, n.d.). Songbirds can vocalize with a syntax as sophisticated as humans (Berwick, Okanoya, Beckers, \& Bolhuis, 2011), a rarified level of synesthesia, but conceptualization is less able in many ways due to smaller brain size, so the range of possible meanings lies far below that of human beings, in reasoning adeptness as evinced by inferior technical skill along with a likewise limited richness of figurative and technical idiom.

Dolphins have brains of similar size to humans with excellent acuity for interpreting the environment, while seeming to share social bondedness and a sense of identity, for researchers note that they call each other by name. Their chatter also evinces complex syntax, with a hefty array of phoneme like sounds strung together in complicated sequences, up to a mind-boggling couple thousand clicks per second (Whitaker, 2018). Social selection for speech like communication in close-knit dolphin pods approximates that of human relationships, as does dolphin awareness of intentionality in oneself and others, cognitive keenness, and libidinous proclivity for vocalization. Highly socialized language is a key aspect of dolphin behavior, and intelligence as displayed by the speed of their conceptual associating is much like Homo sapiens, yet our species arose and developed in a much different environment with a vastly discrepant body plan. Why then did human speech, the first crude traces of which arose later than a few million years ago as the hominin mouth and throat became gradually reconfigured for articulation, join this exclusive club?

In order for a language to contain lots of precise nouns, the creatures which employ it must of course attribute meaning to a large quantity of discrete phenomena, and for organic life this meaning will necessarily derive from the perceived causality of these phenomena as contained in immediate appearances, orientations to bodily form, and relationship to the mind. Sloths move very slowly and are not one of the most intelligent animals, but gravitation-induced challenges of tree environments sculpted their comportment for clutching with a sure grip, albeit at a relaxed pace, so these species have a sense for structural properties that is similar to humans while lacking comparable conceptualizings of them (Worldlife, n.d.). Squirrels are critters with kinetically faster nervous systems tailored for exquisite coordination alongside some technical aptitude in nest building, but their mouths are the libidinous locus of object manipulation and they have small brains, so these animals spend most of their time scrounging around for nuts and other foods to no avail (Bosak, Moore, Masino, \& Klein, 2013). Songbirds are quick-reflexed and more social than squirrels, be vying into large flocks and engaging in humanlike linguistic expression while also having the ability to construct nests, but their brains are likewise small, with attribution of conceptual meanings to large quantities of objects and additional phenomena quite limited. Humpbacks are intelligent and linguistic animals, but as large $\mathrm{Ba}$ leen whales they have almost no impetus to make fine distinctions between ob- 
jects in service of prehension, feeding or rapid mobility, so their majestic songs are slow, protracted affairs, more a libidinous self-expression than analytically aligned to concretions (Editors of Encyclopedia Britannica, n.d.). Dolphins are much faster and socially tight-knit than whales, with a greater amount of their metabolism directed towards fairly large brains, resulting in an unusual level of cleverness as well as high-achieving language ability. However, dolphin physique and way of life do not require the kind of object manipulation that humans, songbirds or even squirrels perform, being centered around merely seizing food with the mouth. Echolocation no doubt gives them good discrimination of particulars, which probably co-selected for assigning basic conceptual labels to what surrounds them, altogether placing these animals closer to humans than most species on the language spectrum without achieving an escape velocity of technicalized memetics like Homo sapiens. It is only in human beings that metabolic primacy and size of the brain, an ultrasocial lifestyle, selection pressure for the cognitive quickness necessary in fluid speech, and manipulation of objects in minute ways via the hands all converged such that conceptually-driven expression became part and parcel of a facility with tangible concreteness that is transforming the world technologically. The question then is how our singular linguistic/conceptual capabilities, provisional of civilization, came together.

The common ancestor of humans, anthropoids and primates was a non-syntactically vocalizing species of much less intelligence, closer to a small monkey than an ape or our own Homo genus. Some precursory factors that would template the human mind include enlarged size of the head and body, allowing expansion of the brain as well as safety and competency for recreational thinking in a greater range of environments, an omnivorous, relatively high protein diet which fueled more metabolically demanding cognition, and cooperative socialization channeling increased conceptualizing into communicative behavior. These dynamics are found in many species besides humans, and of course few of them are anywhere close to linguistic, though most do make meaning-laden utterances. What separates human morphology from the majority is motor coordination for grasping objects, the outcome of ancestral tree dwelling over the course of many million years. As the primate lineage that would become anthropoids bulked up in body mass and, excepting the gibbon line of descent, transitioned to a more land-based existence, mutating physiques were gradually streamlined for a division of labor between walking and handling items with the forelimbs. Humanity's Homo ancestors must have adapted well to covering long distances while carrying loads, for once bipedalism began to take root, evolution only accentuated it. Intention, communalism, technical problem-solving, commuting, bodily function in general were oftentimes absorbed in acquiring, manipulating and sorting items with the hands as a matter of necessity, preference and recreationality, so that language would co-evolve with both a strong sensibility for tactile structure and propensity to inquisitively scrutinize visual details.

What would become human language began to coalesce as utterance was in- 
fluenced by the growing collection of closely inspected, intricately known objects, producing discrete sounds we call phonemes which more consistently and then systematically designated particular phenomena. A plausible hypothesis is that phonetics were molded by facets of underlying protologicality, a linearity of thought that seems common to all especially linguistic species at the least, most likely originating far in the past, perhaps before extinction of the dinosaurs. Collections of particulars and their specific features recognized by the Homo genus increased in breadth, alongside gains in intelligence which made interaction with environments more technological as hominin minds piloted bodies to engage in form-giving behavior for the sake of crafting and utilizing objects as tools, so that technical insights, conjoined with protologicality, enriched and resolved thought into a uniquely precise and concretized kind of self-expressing introspectiveness. It is at this point that advanced conception of the Homo genus started to exact selection pressure on vocalization to assume greater structure as the syntactical permutations of grammar like speech, a period associable with the language-sufficing throat which may have developed sometime between Homo habilis' origins and the Neanderthals (Rice.edu., n.d.).

So as phenomenal attributes of perception became more and more subject to protologicality of both linear and structuralizing types, the nature of introspection seems to have shifted somewhat from stream of consciousness and towards the self's deliberate reasoning responsible for managing technical behaviors and social meanings. Apparitions of the qualitative mind could be better harnessed for practical purposes, with imagination waxing increasingly organized, capacitated to analytically observe, define and modify environments to suit its ends. Blending of incisive protologicality into phenomenality strengthened the cognitive complexing that conceives relations of cause and effect with independence from anchorage in the directly inspected world, and elements of mind which envision pure possibilities and nonexistent entities began to carve out a cognitive domain of hypothetical concepts via runaway introspective imagining. Conditions of leisure won by applying surpluses of problem-solving perspicacity synergized with this reflection, providing greater opportunity for the self to ideate unincarnated, unsubstantiated and speculative realities by thinking, sometimes intermingling these conceptualizations with the apparent environment. Libido was channeled towards creativity and goal-setting behavior by a physiologically imperious consciousness.

At first, upon the origin of the Homo genus and its spread to all corners of the Old World, the burgeoning of this creative intelligence seems to have been directed primarily towards problem-solving for purposes of nutritional needs and expanded range. Homo species besides our own left less evidence of artistic refinement and deeply symbolic tradition, though Neanderthals appear to have produced rudimentary art and carried out burials, but as early as Homo habilis the remains of camps show steady improvement in the quality of tools and additional artifacts related to food consumption (April, 2013; Pobiner, 2016). The 
Homo genus' adaptive radiation conquered every ecosystem encountered with ease, for paleontology of coeval species reveals a large decline in biodiversity, probably due to non-sustainable hunter-gathering and displacement (University of Gothenburg, 2020). Long before Homo sapiens, the genus' libido was being diverted into a highly intelligent cognition of much plasticity, for in roughly two million years around 9 Homo species had protoculturally adapted well enough to achieve cosmopolitanism in Africa, Asia and Europe, while recovery from the corresponding bottleneck by a small selection of other organisms, likely due to rare endowments of intelligence, size, speed, lethality, remote location or some other means to elude, resulted in much more speciation. 38 species of big cat maintained from six million years to present; wild dog populations rebounded to 36 species from two hundred thousand years ago to modern times; there are 29 species of the four thousand year old wild rabbit worldwide. Monkeys, which did not occupy as many ecosystems as our genus and may have been decimated by humanesque hunting in some locales along with ice age shrinking of jungle habitat, boast a whopping 260 species, the legacy of a fifty million year past.

Highly conceptual, all-purpose hominin speech as utilized in especially communal settings gave rise to two important evolutionary consequences. First of all and most obviously, the slight deviation of behavioral impetus away from both instinctual drive and attachment to environmental triggers such as predator/prey dynamics, territories, stringent mating rituals or kinship-centric imprinting, and into concept-based communication of imaginative, innovative kinds, rendered protoculture more subordinate to the self. Slackening of the impositional force exacted by collective norms, which in most species rigidifies cognitive complexes of compulsivity, recognition, intention symbolism and status-related restraint, empowered individual thought processes and identities to sublimate towards freer expressiveness, more reflective crafting of one's personality, and acts of social architecturing. Our genus was becoming able to conceptualize affect, motivation and nature beyond the boundaries of long-standing conformity, a more self-evocative experiencing of the ecosystemic, social and personal, which transmuted feeling into desire, reasoning into a stable fund of analytical knowledge, and customs into a forum for psychological actualization.

Second and more speculatively, it seems plausible as a hypothesis that various aspects of protologicality began to integrate via synesthesia, leading to new noesis. Technological applications had reinforced structural thought, the intuiting of causal relationships between objects, and linear thought was likewise heightened by protolinguistic vocalizing, giving this sequential kind of reasoning more palpable particularity, at which time these cognitive aptitudes synthesized into a kind of mentation that is uniquely human compared to other extant primates, what we know as abstract reasoning. Chimpanzees readily figure out how to assemble complex sets of objects for practical purposes, and songbirds can string together long, highly organized trains of symbolic sounds to convey concepts, but the human mind deeply hybridizes these aptitudes, contextualizing 
structure such that certain properties we typically ascribe to objects-length, width, depth, size, weight_-are conceptually manipulatable by extrapolative inferencing, like the signs and symbols of our infinitely generative verbalizing. Humans not only recognize actual and potential patterns in phenomena, problem-solve creatively using an acute protological sense for objects, and infer meanings with protological linearity, but go further by inferencing about structure with a flexibility that the rest of Earth's organisms seem to not even approach, throwing off constraints of perception as bound to matter in a purely conceptual matrixing of the phenomenal, scaling up or down in great detail, analytically grasping how any form of particular or set of particulars leads to, implies or coheres with others in a disembodied, nonfinite dimensionality that is highly operationalizable but which does not actually exist beyond the mind. This relatively late phase of cognitive evolution made possible both prehistoric and historic construction of epistemes from cumulative experimentation, also the logistical management of civilization, abstract geometry, Platonic-style notions of form, metaphysical and natural philosophy, conceptualizing of the infinite, quantitative theorizing, and ultimately the empiricist rationality of our contemporary age.

What is unequivocally cultural originated with Homo sapiens roughly one hundred to two hundred thousand years ago. Archaeology of early human settlement displays unmistakable signs of our own enculturated psychology: meticulously designed implements of much variety, ornately decorative art, and artifacts for spiritual ritual, analogous to those of anatomically modern hunter-gatherers as recorded in the documentation of many civilized Westerners (Erard, 2018).

How exactly humanity's ultra cultural practices evolved is not simple to apprehend, but conjunction of imagining the nonexistent, conceiving objects and phenomena in general within contexts of abstraction, and trajection towards more flexible normalization in societies offers some respectable explanation. Higher technology can be accounted for as a projection of inventive abstraction into our interactions with the environment, a creative structural inferencing which together with malleability in cultural normalizing countenanced transmission, progressiveness and adaptation of technical customs to a gigantic array of circumstances, eventually modified for the sake of densely populated civilizing. Spirituality and mystical ritual clearly have their roots in the imaginative and often fantasy-driven interpretation of enigmatic, incomprehensible or uncanny phenomena, spun into traditional myths diverse as human culture itself. We can understand artistry as taking shape from out of a similar psychical wellspring as technology, being the aesthetic and symbolic dimension of technical functionality. Blossoming of the human psyche in more permissive, less instinct-governed and hylic-oriented environs contributed at times to inspired, highachieving states of mind, while also ushering in norm-bending deviancies of motivation and temperament which leads to all kinds of self-destructive and disturbing behaviors even today, what is conventionally adverted as madness or 
evil. The human psyche is an amazing entity, difficult to fathom, stretching much deeper and wider than conception alone, but to the extent that the species' minds have pragmatic objectives of any explicitness, our intention-driven reasoning is foundational in sustaining the long-term direction of an individual's decision-making and also the most critical factor for any civilized collective's fate. Let's take a closer look at the anatomically modern human thought process as well as its alignment with and impact on psychology and culture.

\section{The Evolution from Precivilized to Civilized Human Conception}

At the boundary between the mind's qualitative states and what we model as corporeal "matter", a congress takes place between organs of sense-perceptioneyes, ears, tactile nerve endings, nose, tongue-and aggregate mass comprising our planet's biologies and ecosystems. While these components of perception are not utterly universal, for pathologies of sensation such as blindness, deafness, numbness, or deficiencies of smell and taste of course occur in a proportion of individuals, as well as much subtle variation along spectrums of sensitivity and quality of sense-perceptual experience, the portion of environments which sense organs are addressed to has been extremely uniform over the course of Earth's evolutionary past, with only brief interruptions of cataclysm for hundreds of millions of years, as well as far and away the principal criterion for survival of our bodies. Thus, sense-perceptual content anchors objective practice in association with a complementary paradigm of mechanistically material, "physical" knowledge, the standardized empiricism and education responsible for guiding civilization's development, in medicine, technology and elsewhere.

But the domain of phenomenality we recognize as "consciousness" of course reaches far deeper than mere sense-perception, for cognition is a rich tapestry of much and typically all of the following: mental images, audible pitches, odors, gustatory experiences, feelings of objects and additional phenomena as well as visceral awareness of one's own affective states, also an orienting of both the mind and body to their surroundings, all of which can be recapitulated in memory or imagined while entirely lacking sensory stimulation. Conception is a further layer of the qualitative, involving thought processes and intentionality, a sublimating of phenomenality into meanings exacted behaviorally and conveyed communicatively.

The multitudinous forms that constitute experiencing are not absolutely delineated from each other, but include much integration by way of synesthesias and more mechanisms coordinating function. Many human beings vividly see sounds and numerical concepts, intuit objects, concepts, the flow of both music and language as evincing an intrinsic logicality, and feel a wide assortment of qualia in nuanced ways. Sometimes a mind loses touch with reality, when it hallucinates, is swamped by strong affect, or misapprehends the causal implications of events. 
Theorizing all of this interwoven diversity seems daunting; is it even possible to categorize human thought and its relationship to broader consciousness? A comprehensive definition of psychical structure is improbable, for even if we arrived at a model encompassing the entire comparative anatomy of human cognition by extremely advanced science, we would still only be able to account for the import of novel mutations using some kind of experimental trial, perhaps necessitating such fine, instantaneous adjustments that any realizable modeling falls short of perfection. And if anything approaching this level of comprehension is attainable, it would require millennia of research within a culture superior to our own both logistically and ethically. However, some preliminaries are accessible based on current knowledge, which may not in itself give us maximal certainty, but nonetheless helps by depicting our body of fact in a consolidated way so we can better grasp the whole and more strategically orchestrate all kinds of episteme-building investigations.

When analyzing the nature of our cognition, it is hard to know where we should begin, but as has been mentioned, songbirds show much likeness to humans: grammar, concepts, fast neural kinetics, some structure-building aptitude, and sociality. These small, culturally inconsequential birds are almost a microcosm of human nature, containing many of its elements in a simplified form that is easier for theory to contend with, providing us a sort of schematic from which the general picture can be elucidated.

Songbirds have well-developed eyes, engage in communication of a grammatical nature which necessitates sharp hearing, grasp with their talons in a similar mechanism to human hands, and have fast proprioceptive reflexes so that they can rapidly maneuver in flight as well as fluently carry out vocalizations. This is much like human beings, differing more in degree than kind, for our cognition performs all of these functions but on a larger scale due to bigger brains, perhaps minus neural speed necessary for navigating the three dimensional complexity of trees and staying in constant motion to avoid predation.

Like humans, songbirds have facility with structure concepts, for they erect nests that are intricate masses of sticks and brush, clearly envisioning how parts fit together as a whole. Beavers display a similar behavioral repertoire when they build dams, squirrels as they construct their abodes, and even though many of the more highly cognitive mammals have much different ways of obtaining shelter, perhaps merely digging and adorning a hole in the ground, the adaptability each of these organisms have to differences in time and place entails at least rudimentary kinds of protomechanistic reasoning from novel cause to imagined effect. In what measure this springs from linkages between cognitive centers of structural and linear protologicality in other species besides humans is mostly unknown, but as has been described, if there is any analogizability it is obvious that humans are superior in this regard. Our species adapts protologically structural thinking to vastly more and larger scale contexts, while humanity's abstract inferencing in the domains of both sign and image symbolism is more capable. Even children of average intelligence catch on to the infinitely recursive 
nature of numerals after exposure to sequences of only dozens of numbers (exceed ten in a base ten system and they begin to get it, infinity), and have no trouble incorporating these linguistic like concepts as quantitative labels for the proportions and additional properties of basic shapes. Chimpanzees, our closest relatives, can do a respectable job of object manipulation as adults if utility for behaviors such as food-acquisition becomes apparent, but applying abstract signs to figures, then deriving mathematical principles according to which these figures are systematically permuted in infinitely flexible ways, a purely conceptual language of objects, is as everyone knows completely beyond them.

While we observe songbirds, it is noticed that their movements tend to happen in bursts, quick alternations between full discharge of kinetic libido and pausing. Their jerky proprioception and perpetual activeness are a stark contrast to human behavior, as our motions show greater fluidity, much less explosive, while periods of inactivity are more contemplative, during which libido is channeled into the intricate processes of cognition more independent from environmental cues. When we compare these small songbirds to not only humans but larger mammals in their entirety, this seems to suggest a general principle approximating the nature of cephalization quite well: the larger a species' brain is, the more potential for differentiation into functional subcomponents together with possible factors of neuromaterial interactivity, instating a higher ceiling in the ambit of coordinated sensory recognitions, qualia, affect, concepts and thoughts. Even when species with larger brains are not extraordinarily clever from the standpoint of reasoning or communication alone, they are likely to possess a more self-regulating holism of consciousness, the precondition for diverse and subtle comportments such as an emotional life to perhaps evolve. In the descent of anthropoids from primates, this cohering of multifarious brain regions trajected towards increases in what we know as self-imaged intentionality, a concept-driven personal and social identity. In our own Homo lineage, procession towards purpose-driven existence reached full throttle, as these species were strategizing in ways that drastically changed ecosystems on every available continent within not much more than a couple million years, barely a blip on the evolutionary timeline. Anatomically modern humans have arrived at an advanced enough stage in conscious control to utterly remake much of the planet, as many of the species' members manage to aim deep and sustained thoughts at unprecedented problem-solving for the sake of labyrinthian actualizations.

So in summing this along with some content from the previous two sections, it seems possible to divide human cognition into three categories. First, there is perception, the qualitative endowment of sensation (exteroception), qualia, proprioception and affect, a general profile which humanity shares with the majority of species, but of course our own incarnation has its modicum of uniqueness. Then we have structural protologicality, intuitive notions of particularized form that allow numerous species to more potently harness their perceptual states for utility's means and ends, with our genus achieving a sophisticated level as it grew more technology-oriented. And finally, linear protologicality grants all kinds of 
organisms the proficiency to execute reasoning sequences, enriched in humans by ties with evolving vocal communication into an autobiographical, self-defining introspectiveness and bent for analytically inferencing at length. The human synesthesias that integrate these domains are no doubt complicated, but quite subjected to the self's intentional thinking, which in turn organizes and directs disparate cognitive processes towards particular goals streamlined by behavioral traditionalizing in consort with environments. The pragmatisms of studious observation and thought as born upon by evolutionary circumstance tend to exert selection pressures on parsing and recombining physiological centers of perception, structural protologicality and linear protologicality towards three gravitational poles of functional synthesis parameterizing the spectrum of intentional thought, altogether the three main types of conception.

Perceptual and structurally protological processing conjoin in structural conception, the mentality that envisions how phenomena fit together for humanity's elementary technological purposes. This type of thinking is the basis for hominin and early human fabrication of tools as well as the construction of simple living spaces, where all requisite features are tangibly present to the mind, which must modify and place them into the desired form by procedural increments. In modern life, structural conception takes center stage when a purchased product requires assembly, also as appliances like vacuum cleaners and computer interfaces are used, or while operating heavy equipment such as a lawnmower or forklift as well as more complex machines such as cars and planes, together with any instance where gadgetry needs repair. It makes its presence felt in art as creativity submits to formal conventions. Essentially, this is the mind organizing phenomena, with strong attachment to objects concretely handled and imagined as constituent particulars, by conceiving their correspondence to proprioceptive and mental routines that will place them in emergent order.

From perceptual and linearly protological cognitive processes we get expressive conception, the employment of phenomenal content to convey intentions as a sort of narrative generated or embedded in the world around us. Its human form originated from out of spoken communication's nonliteral facets, language's multiple layers of meaning contained in imagery, metaphor, and symbolism of expression generally. With prehistoric societies, this way of thinking was closely related to spirituality, myth and the enigmatic qualities of motivation, a factor in humanity's compulsion to formulate epic stories, allegorical dances and additional enculturations, which lended comprehensibility to deep-seated drives, humor, mysteries of the largely unknown and undomesticated world as a whole, a cerebrally satisfying, higher functioning coherence. Modern art provides an example in the free verse poem, evocative of impressions, struggles, beliefs and values, a culturally condoned mode of expression that contains ambiguity and profundity of peak nonliteral intelligence while minimally intermingled with structural formalism like we find in genre-bound literature such as 
Shakespearean poems, romance novels and all kinds of additional works.

Structural and linear protologicality bring forth iterative conception, already presented as the means by which humans inference extrapolatively and interpolatively, reasoning from particulars towards generality that is not affixed to the particulars themselves as their latticed discreteness makes its appearance in nature or otherwise manifests to the mind, a cognitive process we commonly call "abstraction". This is a kind of thinking that deduces within the realm of infinite possible form, permitting us to manipulate concretions far beyond the constraints of immediate perception, a cognition-centric palpability of pure ideas and their instantiations in hypothesizing. Prehistorically, it took analytical adeptness to the next level, for humans escalated from apprehending causality within spatiotemporally localized contexts of object utility, such as in the case of handheld implements or simple huts, to deriving holistic models integrating total reality, doggedly progressing a fund of knowledge and practice by revisionary experimentation, building more and more of the environment into encyclopedic compendiums of descriptive representation within the contexts of an unbounded dimensionality. Ancient humans began experimenting with ecosystems, working out selective breeding and land use, constructing planned towns and cities in the first civilization, also immortalizing natural patterns and the most prominent events using record-keeping systems such as calendars and writing, refining the diverse disciplines by which technical methods are developed. In contemporary society, computer programming is a perfect example of iterative conception: coders obtain an idea of what they will strive to create on the monitor, then tinker with detailed object language systems in order to embody the systematic objective in hardware, making copious corrections during moments of error by sequences of deductive troubleshooting until design is actuated. In terms of institutional practice, science is a pinnacle of iterative thought, advancing our theoretical and technological paradigms by an effectively limitless accumulation of fact-based inferencing and discovery.

These three types of conception all have much importance from a cultural standpoint, but are also simply an inherent aspect of ordinary life. Human nature is stimulated towards structure-building, meaning-saturated expressiveness and iterative problem-solving every day, an impetus ingrained into the very core of our existence. Conceptuality is a run-of-the-mill given, but due to the highly self-aware and socially conscious makeup of human psyches, our thoughts and behaviors are also deeply symbolic of who and what we are to both ourselves and those around us. Of course conceptualizing must not only represent who we want to be, but just as much if not moreso what those around us as well as subcultures and institutions expect us to be, for which reason identity projection with its long-term ramifications for an individual's fate, including basic quality of life, is a delicate matter, requiring great care if we are to navigate society.

We are constantly giving demonstrative shape to experiences in social settings, often not that profoundly or provocatively, yet situations sometimes compel us to seek optimal performance for the sake of achievement: a career-defining 
interaction at work, a make or break moment in some personal relationship, any time and place where showing off our most impressive talents is of benefit or called for. Usually even high-functioning actions are moderated by collective normalization, but in art and elsewhere humans on occasion prevail against all odds in throwing off the shackles of a persona we all must craft as social compromise for the sake of communality, at which time a singular best we usually feel the obligation to conceal is unveiled, bursting out of normalcy in peak spontaneity, creativity, intelligence or athleticism.

Enculturation of high achieving conception is currently impossible for the single individual to exhaustively theorize, for each act or social movement has a daedal host of contributing factors. This is material for the most skilled historians to research and write volumes about, for the truth is much vaster than the whole of apparent reality as it presently stands. As for how ingredients of human motivation fit into the picture, we can make some generalizations: accomplishment is sought for personal and cultural accolades; as an actualization of one's nature, identity or ideology; in order to give and receive pleasure; for its logistical practicality; and in order to emblematize standards and values. Culturally significant products of conception are incredibly varied, whether technological, artistic, and private or of mass appeal, but all symbolic of both particular creators and the societies they inhabit, flowering from out of biologically rarified ambition and an often spectacular inventiveness to amplify life's meaning. The most eminent human feats employ structural, expressive and iterative thinking all at once, but usually with more emphasis on one or two of these three types of conception.

Early hominin technology placed greatest weight upon structural thinking, a largely function-oriented concern for the ergonomy and durability of constructed objects. The psyche evolved towards greater depth in conjunction with enriching funds of meaning, largely inspired by language use, and humans incorporated more and more expressive symbolizing into design until memetic content was integral to technology. By the time our species had entered into civilized living, a specialization of nonlinguistic art-painting, sculpture, architecture-was subordinating structural concerns to expressive symbolism, with this craftsmanship and its growing cultural force as much about aesthetics as the practicality of form. Of course expression via structure prompted iterative thinking also as artists experimented with techniques and styles in order to advance genres and quest for originality. In the 20th century, extravagant iterativity of a lifestyle based upon science and electronics became not just implicitly invoked by method but an overt feature of visual art as prominent works embraced, rebelled against, came to psychological grips with a world in which the future will be determined by computationally inferential form.

Expressiveness of the human psyche has its roots in ancestral species' aptitude for symbolic recognitions, evinced by thousands of additional biological lineages as well. Even moderately advanced cognition can experience phenomenal attributes as symbolic of causal properties in the environment, learning, predicting, putting 
two and two together by inspection of indirect evidence. Organisms pick up on each other's scents, tracks and sounds, from which is constructed a mental model of behavioral tendencies, whether for hunting, eluding predation, or seeking a social opportunity. Likewise, weather and the body's homeostatic states signal seasonal exigency, inducing activities such as migration and hibernation that are carried out with greater success when an animal's thinking is more capable. To illustrate this, we can simply compare a Monarch butterfly to a grizzly bear: these butterflies manage to migrate thousands of miles, completely beyond the capacity of a bear species that, for analogous purposes, can do no more than hole up in the vicinity, but the ascendance of grizzly intelligence was such that this animal became almost impervious to death by starvation, violence or other non-natural causes, its food-finding and danger avoidance rather cunning and resourceful, while thousands upon thousands of Monarchs die each year from a relative absence of foresight (The National Wildlife Federation, n.d.a, n.d.b). In general, an individual mammal's prospects prove better than an insect's with its much smaller brain, for implications of environmental patterns and perception generally are in the former case more interpretable.

The primary precondition for graduating from recognition of attributes as symbols to symbolic expression is robust intentionality. As has been mentioned elsewhere, intention evolved as mode-selecting awareness for internal control of brain states, empowering the mind to align with environments in more context-sensitive ways while also placing further checks on the reflexivity of stimulus/response, beyond simple sensitization and habituation, so that delayed gratification in the service of more efficacious outcomes became possible along with diversification in the repertory of behaviors, increasing adaptability of individual organisms to nuances of circumstance. Attention span and improvisational thought advanced in some species until a sort of primordial introspection arose, which assessed cause and effect entirely absent environmental cuing, by self-directed conception conjoined to perceptual stream of consciousness.

In most natural settings, selection pressures are exerted on the problem-solving self to target wholly practical objectives, whether of feeding, mating, sheltering or safety, limiting the creativity of most organisms. This is clear from observation of how vocalizing bird species have a more economical range of calls when their lives are spent in the wilderness, deprived of the ample food and relative security that can be afforded by close contact with humans. Cognition in these cases is honed for a lifecycle overridden by material requisites, with libido canalized towards functional need. When introduced to captivity, provided that basic essentials are readily accessible and stressors as well as other preoccupations minimized, many of these birds start to sing more inventively, as if entertaining themselves during idle stretches by novel riffing (Williams, n.d.). We of course see the same phenomenon in our pets, albeit often less related to conceptualization: when certain dog breeds are left to their own devices, they incessantly chew for no purpose but recreation; some cats will paw a toy mouse around the room 
and repeatedly pounce to mimic the pleasure of hunting; a hamster has great fun mock scurrying on its wheel. Offering pets diversions that have no problem-solving stipulations places little strain on their cognition, so that domesticated recreating does not perforce incline towards extraordinary intelligence, but in order for a wild animal to come upon the same level of idle time, it must be smart enough to have mastered its environment. There is much besides an organism's wits that figures into this type of behavioral supremacy, such as sparsities of both threat and deprivation due to size, speed or group congregation, but when some or all of these factors happen to intersect with introspection potential, the devotion of libido to self-amusement of imaginativeness along with physiological dynamics such as neoteny (Rice, 2011; Bond, 2020a) can select for the evolution of an identity-complex in the organism's mind, a self-awareness constructed from keenly observing and reflecting upon its own experience.

As previously discussed, the Homo genus was quite sophisticated in this respect, harnessing nature in unprecedented ways with technological insightfulness. Consciousness in these species was becoming able to discriminate more obscure relationships between many kinds of phenomena by introspection-informed observation of perceptual patterns within the scope of its structural protologicality. Hominin minds simultaneously moved ever closer to resolving linear proto-logicality into the thought process we know as logical inferencing, which would one day interface written symbolism and structural abstraction within a culture of rationalist empiricism in order to disseminate high technology worldwide, thus far the apex of humanity's competency for analyzing and utilizing environments. But before all of this possibility could be realized, the human race had to evolve its language faculties. What follows is a series of plausible hypotheses about how this process unfolded.

A key factor must have been evolution of brain regions that interface cognition with vocalizing for the sake of articulated utterance, what we know as speech. This mental scaffolding that fine-tuned unconscious processing, intentional thinking, the forms and modes of meaningful statements, and facial coordination to complement each other during acts of verbalizing is of course exceptionally versatile, adopting a plethora of configurations depending on expressive context, the heterogeneous reality of which formal grammar and analysis of logical argument do not even begin to capture. At base, this structural parameterization is made up of an intuitive grammar roughly divided into conventional parts of speech with very flexible attachment to meaning in many cases, and a sort of expression-centric protologicality, distantly approximated by the basics of formal logic. Theorizing these underlying structures calls for punctilious research on a level that linguists have probably not yet even dabbled in, a task for science of the future.

Individual and relationship psychology likely contributed to the evolution of language in multiple ways. First, motivation to vocalize is of course necessary, a characteristic shared with thousands upon thousands of nonhuman species. The 
Homo genus must have begun reflecting on its own vocal behavior as it became more introspective, resulting in primordial cognizance of utterance's structure and eventually an awareness of expressive sound as involving something like technicalities, which caused the patterns of utterance to grow more consistent. Conceptualizing of utterances as a sort of phenomenal object and then a construction took hold, so that articulation acquired greater aesthetic impact, with more pleasurable, skillful, difficult and beautiful expression held in higher esteem, impressions no doubt stimulating much mimesis in prehistoric clans and tribes. At this point, two threads of evolutionary development must have been in effect: the most functionally and aesthetically popular of these species' expressive tendencies unfolded in a train of progressing social conventions, advancing language as technological and artistic protoculture, while any mutations conferring superior ability would have quickly improved language via mimesis. Thus, reflective observation, aesthetic sensibility, cognitive mutation, imitation and protocultural traditionalizing moved the Homo genus towards linguistic communication, a behavioral trait that is crucial to anatomically modern Homo sapiens' higher cultures and which likely played a main role in bridging the gap to our more expressively symbolic ways of life.

The first semblance of human language was probably short declarative statements, then rudimentary conversation which hominins and early Homo sapiens took part in primarily as recreational diversion. With humans at least, expression became elaborate enough in its structure to permit storytelling, and the constructing of narrative is of course a core feature of not just casual but more ceremonious forms of socializing, with many prehistoric and historic tales alike serving as culture-defining myths, ritualistically retold, reenacted, shared for millennia as part of basic public consciousness. At the same time as intention to express oneself and the values of one's culture molded verbalization, speech acts likewise selected for the structure of thought. Linear protologicality of the introspective mind grew increasingly organized while it interacted with linguistic behavior, perceived more and more as chains of syntagma within definite yet infinitely generative meaning. Open-ended iterativity of narrational sound coevolved with a knack for iterative conceptualizing, the apprehension of languagelike sequences and further arrangements of symbols in the form of inferencelike abstractions and eventually infinite schemas. This affinity for the abstract ultimately prompted humans to invent writing systems, a seminal method of civilization.

One of the significant benefits accrued from linguistic behavior was flexibility in the boundaries of social relationships. Full-bodied language made thinking of almost any complexity or novelty provisional of being expressed with explicitness, while generating conditions under which unprecedented thoughts and behaviors are admissible. Human bonding does not merely rally around recognition of obvious means by which to satiate drives, such as in hunting, self-defense, mating, familial caretaking or additional compulsive activities, in essence crude need, but conveys concepts and reflects upon the insights of fellow individuals 
via the medium of language in a cerebrality and tolerance for comprehensional obscurity that is probably almost unparalleled by organic life on our planet. Even the most arcane experiencing can diffuse into the cultural milieu as humans attempt to express unconventionalized and even nonfunctional ideations, with brute negative feedback attenuated by the intellectualized prerogative of discoursing, so that groundrules of mutuality do not inhibit the independence and diversity necessary for higher level reasoning. Humans are supremely innovative while nonetheless managing to subsist in extremely normalized, eons-old communities.

Convening the whole of human cognizing towards collective purposes succeeded in tightly binding individuals of prehistoric clans and tribes on numerous planes: members of our species were not only drawn together by feeding, reproduction and protection, but also from out of more conceptual communality such as shared beliefs, spiritual and symbolic rites, gods, technological methods and inventiveness, rituals of many kinds, conversational fraternizing, context-variant manners and mores, all inculcated by way of teaching, learning and reflecting over the span of centuries. This arranging of human life by precocious cognition kept tribes close-knit even as languages and traditions underwent evolutionary drift, which was a huge boon to in-group solidarity, but also a driving force for the rapid divergence of separate cultures, so that when communities lost contact, they could arrive at discrepancies in conceptualizing, expression and practice bordering on incompatibility within only several generations. This was a blessing and a curse, for human decision-making and behavior are massively adaptable, but we can tend towards misunderstanding, obstinacy and confrontation during intergroup interactions.

Maturation of both language and symbolic culture commenced the prehistoric stages of anatomically modern human progressiveness, probably beginning its development at least a hundred thousand years ago (Linguistic Society of America, n.d.). These endeavors often synthesized three general categories of focus. Techniques of structural artistry played out in every culture, including ornately designed buildings, pottery, as well as all sorts of weapons, tools and additional implements. The chiefly structural and expressive thinking required usually demonstrated some primitive iterative thinking as well, for most decorating of objects and constructions included strings of geometrical shapes or nestings of shapes within shapes, similar to the kinds of images that might be yielded by the recursive protocols of a simple computer program. Deference to the spiritual also held a prominent position within ancient society as it still does for many today, an awe and wonderment at the supernatural, tapped into by mystical and religious practices. Self-iterating experimentalism also played an enlarging part in human lifestyles, the tinkering adaptation to environments throughout the world, assaying manipulation of natural phenomena with a flair for protologically deducing relations of cause and effect, while also showing the first glimmerings of more conceptually advanced sorts of correlating within the arenas of planned 
ecology and management of trait heritability in Earth's organisms.

The most ambitious exploits undertaken by our species were sometimes ingeniously successful, but occasionally real head-scratchers. Evidence suggests that the Amazon rainforest was cultivated by prehistoric humans, a very early and amazing achievement in the domain of ecology (Mann, 2005). In remote parts of central Eurasia, huge expanses of buried bones and additional paraphernalia have been discovered. What possessed precivilized humans to gather for the sake of depositing remains and artifacts into areas the size of a football field is hard to decipher, but it seems thousands of individuals must have assented to the practice (Torrey, 2017). With a variety of outcomes, it is clear that even prehistoric humans were mastering every geography on Earth, imposing cultures of not just functional creativity but protoideological purposes of colossal scope.

Humans embarked upon civilization around 10,000 B.C.E., living a life centered on agriculture, animal husbandry and town dwelling, with settlements springing up everywhere, linked by bustling economies of trade (Pearsonhighered. com., n.d.). Technological and artistic advancement certainly took place over the millennia, but the biggest transformation occurred a couple centuries prior to 3000 B.C.E. with the invention of writing. It originated as a derivation from pictorial art, using images to represent concepts, with various systems tailored for different contexts. Over the centuries, writing techniques were improved until more abridged symbols for numbers and verbal expressions came into usage. By 1000 B.C.E., an alphabet of symbolic letters closely aligned to the phonemes of speech had been contrived by the Phoenicians, which quickly spread in its essentials to other parts of the world (Schmandt-Besserat, 2014).

In addition to literary labors, writing was first employed in record-keeping for administrative purposes such as taxation and business. It also found application to more naturalistic avocations, especially in documenting the movements of celestial objects (Whipps, 2008). Rudimentary symbolic labeling of instruments was used for the measuring needed to survey property and carry out ambitious architecture such as temples, palaces, monuments, large-scale public works and city planning (Boyer, 1991). With more settlement, trade, wealth and mobilized manpower, quantitative methods for construction proliferated, ever more comprehensive, birthing an alternate universe of structure concepts and their functions as the strategic core of civilization.

It was at this point that iterative thinking really took off under the influence of multiple factors. Aristocracies gained a lasting foothold as the upper echelon of society, and their financial independence from the occupational grind liberated them to launch adventuresome enterprises. A leisure class of wealthy citizens or those supported by them began speculating into erudite pastimes, self-determination often devoted to pondering abstractly. Interest in literature increased, and this forum for composing one's deepest contemplations in writing stimulated those aspiring towards intellectuality to carefully reflect upon ideas and iteratively revise them in the service of clarity, precision, and analysis by posterity, 
coalescing into a subculture from which the rational ethos and its upgrading of logical and empirical methods would germinate. This was conjugated with venturous contributions of iterational abstraction to civic practices of quantitative conceptualizing, building and artistry, in essence the scaling up of schematics with their mathematical features and an interpolation of symbolic sculpture, painting and additional expressive art into these complicate, compound frameworks, altogether setting the stage for societies of theoretical analyticity.

For the Western world, highbrow culture seems to have found a permanent niche in 7th century B.C.E. Greece and its colonies. While we have little evidence of this movement's premature eras, most of which is secondhand, the many references of antiquity's later, preserved philosophers and historians to schools of thought and their rivalries as well as an occasional homage to primogenitors intimates the timeline of paradigmal evolvement with a fair amount of certainty.

Thales (b. 626), Anaximander (b. 610) and Anaximenes (b. 585) sought to ascertain the natural world's "arche" or essential principle of formation as manifest in apparent substance, a very early instance of philosophical materialism. Pythagoras (b. 570) soon followed with postulation that the substance of nature has fundamentally quantitative form. The Pythagoreans carried on a tradition of austere mental, behavioral and spiritual discipline, some of the first signs of what would become Greek academia's rational ethic. Heraclitus (b. 535) proposed that the natural world's essence is not contained somehow in material substance, but rather exists as a supramaterial impetus of fundamental flux or "becoming". Parmenides (b. 515) championed the opposite idea, that all change is an illusory instantiation of eternal, unchangeable "being", the metaphysical "One". $\mathrm{He}$ founded the Eleatic school; by the 5th century B.C.E., multiple centers of philosophical study were scattered amongst the Greek community, indicating that literary innovation by individuals was fast growing into well-funded, multiagenerationed collectivity, the first signs of institutional education in the West. Democritus (b. 460) set forth a theory that the material world is composed of fundamental particles he called "atoms". Empedocles (b. 434) proffered a complementary theory that the material world's substantiality on the macroscopic level admixtures four basic elements: "earth", "air", "fire" and "water".

It is clear that iterative thinking was elevating in prominence as early Western philosophy performed this progressive series of thought experiments from which were derived increasingly incisive pictures of the world, utilizing extrapolative inferencing from particulars to generalized protomodels. Greek society was building an explanatory edifice of structural abstraction by applying abstract inferencing across large spans of time, and both of these domains, structural and inferential reasoning, were waxing more systematic as methods of analysis.

Plato (b. 428) consolidated the philosophical notionalizings of substance and reasoning that had been under development for roughly two centuries. His metaphysics theorized an eternal substrate of abstract "form" as the essence of both "nous" (soul) and "hyle" (matter), probably with heavy influence from Parmenides, and averred in line with Pythagoras that the Forms are intrinsically 
mathematical. He seems to have embraced the Pythagorean concern with intellectual and behavioral indoctrination, for he used his aristocratic stature and wealth to launch what was probably the first purely academic institution of learning in the Western world. Plato's new Academy inherited the legacy of an Athenian golden age that ended a few decades prior, which had been led by the statesmen Pericles, an expert in oration who committed to furthering the cause of Athens' citizens with democracy-enhancing reforms and lavish spending on civic beautification. Political participation was at its height, bolstered by sophist-guided instruction in law and rhetoric, and Plato exploited this public consciousness quite well in order to promote the Academy, authoring popular literature that would present the philosophical tradition and his own ideas in an accessible way, via the genre of Socratic dialogue. His works display a strong logicality, with reasoning that is nearly formal in its degree of organization, culminating a long history of inferential discourse. Plato's appeals to a general audience are of course extensively intermingled with expressive device, but he also spent some effort promoting what are called the "unwritten doctrines". These were only discussed in lectures, regarded by him as too abstruse and susceptible to misinterpretation for literary presentation, probably groundbreaking for the time. He apparently blew away crowds with astronomy and abstract mathematics, likely siphoning to the conceptual core of his iterative intellectuality.

Aristotle (b. 385), a foremost originator of empiricism in the West, was educated at Plato's Academy in Athens, hired to tutor future conqueror Alexander the Great by the king of Macedon, a principality north of Greece, and served as the director of many academic enterprises. His philosophy synthesizes metaphysics, rationalism and materialism in a theoretical framework for conceiving the relationship of substance's essentiality to fluctuating apparencies in nature. He assimilated Empedocles' schema of four elements, adding "aether" as a type of matter hypothesized to lie beyond the terrestrial sphere. Aristotle envisaged a dynamic cosmos, defining "being" itself as a "prime mover" which shapes reality, including within its scope the reasoning "active intellect" of humans, in accordance with four principles of causality. Paripateticism did not, in the manner of Plato, regard truth as accessing an absolute universality, the essential Form of existence, but stressed essence's multifariousness as manifest in the world of immediate appearances, a more naturalistic emphasis. Aristotle undertook many expeditions to examine and catalog properties of the Aeolian peninsula's plants, animals and geology. He likewise investigated human behavior and society with a greater empirical sensibility, enumerating and explaining the plain facts of relationships, practices and politics rather than promulging lofty ideals. He also introduced a large dose of empiricism to consideration of reasoning itself, fashioning a theory of correct inference known as syllogistic logic. Aristotle set precedents for adapting structural reasoning to the diversities of differing contexts, an impetus for the expanding specialization of scholarship, and transitioned inferential reasoning from collaged rhetorical styles towards an integrated system, sowing seeds of the future's even further methodologizing in formal proof, con- 
ceptual schematizing and technical language, particularly important for mathematics, scientific modeling and programmed electronics respectively.

Alexander the Great's governance spread Hellenistic learning to much of the Mediterranean and Middle East, and a city he founded in Egypt called Alexandria with its renowned Great Library served as headquarters for the empire's upstart scholarship. Euclid (b. 365) moved to Alexandria and availed himself of this city's resources to compose Elements, a survey and analysis of the era's mathematics that would be a preeminent textbook on the subject well beyond the Middle Ages, edifying the likes of Galileo Galilei and Isaac Newton. He seems to have absorbed many influences: a more than two hundred year tradition of formulating the general principles latent in applied quantification, analyses which had been performed by mathematicians such as the Pythagoreans, Hippocrates of Chios, Eudoxus of Cnidus, Theaetetus, and Philip of Opus; Plato's conceptualizing of existence as mathematical form; Aristotelian-style crafting of specialized disciplines as the first academic fields; and formal rhetoric. He invented a systematic format for carrying out deductive proof, deriving an integrated framework of general axioms from basic propositions, and devising a method of problem-solving demonstration for exemplifying the essence of his book's concepts via geometrical constructions and symbolic labeling. It was the most thorough melding of structural and inferential reasoning in the West until the Early Moderns fused coordinate systems with linear algebra in "function"-based geometry, and a paragon of logical rigor.

So there seems to be a close relationship between analytic enculturation and the development of iterative thinking. Prehistorically, accumulating ingenuity for applying iterativity to both pictorial art and conceiving the environment suffused a greater gamut of the abstract into expressive and structural conception, contributing to the development of primitive ecology as well as civic and artistic design. Structural iterativity was adapted into systems of image symbolism for concept expression as the first writing, and then into much better methods which represented the expressivity of spoken language directly, assuming documentational and literary roles. Antiquity's humans worked writing into schematic diagramming in order to platform more complex engineering projects, and also finely sculpted the terminology and reasoning of precision communication via a philosophical discourse furthered by literature. As demonstrated in ancient Greece, philosophy's analyticity and its growing ethos of intellectual integrity became a formidable subculture, with numerous communities and their schools of thought striving towards comprehensive accounts of reality's properties and patterns. Rational collectivity evolved into academies, well-organized enough to formalize the categories of knowledge as institutional traditions concentrated upon strategic acquisition, collation, evaluation and progressing of fact-based theory as informed by abstract reasoning. Theorizing the essentials of theory itself brought more lucidity to structural and inferential reason, birthing formal methodologies of deduction, most notably Aristotle's qualitative logic of 
linguistic truth-value and Euclid's framework for quantitative proof, laying the foundation upon which Western civilization's vast assemblage of rational theses, professional rhetoric, technical modeling, pedagogy, and ultimately high technology would be raised.

As far back as antiquity, it was apparent to some scholars what theory might become: an epistemic fortress incorporating more and more of reality into its sphere of influence, steadily reconfiguring conceptualizations, perceptions and environments into forms with greater amenability for human life, actualizing the species in ways that would be without peer in Earth's history. The foremost challenge of civilization then presented itself, how to make concepts and methods of theoretical knowledge the core of culture.

\section{The Evolution of Intellectualized Conception and Discourse in Western Civilization}

At the beginnings of civilization, humanity's reasoning intuitions were being applied almost exclusively in their natural role, as a means to make judgements regarding the structure of environments for technological and ecological desideration, crucial to selective breeding, construction, management of food production, and settled life generally. Early civilized humans conceived hypothetical solutions to the problems of a more intellectually arduous existence, put these practical insights to the test in daily life, and then carried out modifications based on the results. This amounted to the employment of deduction, an inferencing from conceptual structures to anticipated facts, coupled with induction, an inferencing from observed fact to implications for the viability of conceptual structures, in essence "scientific method", but not yet having been explicitly formulated such that it served as a formal procedure for cumulative progress, still embodied subliminally in technical developments at this stage which were closer to opportunistically conditional than systematically directional.

The first literature of antiquity aimed primarily for rhetorical effects, telling an evocative story and symbolizing the values of one's culture while minimally concerned with technical or empirical accurateness. But as technology advanced, taking center stage in human relationships and the sustenance of civilization, authors began to construe the traditional content of written artistry-historical origins, the nature of the world as a whole, social dynamics, the meaning of human life-as amenable to scrutiny by reasoning. Even the most analytical works did not initially extricate themselves from rhetoric, and combined with the enticement to essentialize, reducing encyclopedic knowledge to underlying intuitions and then erroneously reifying it as reality's absolute principles, nascent philosophy usually emerged from the quest for comprehensive truth with an image of the world as driven by fundamental rationale, a supreme ordering analogous to human law.

In ancient Greece, the fountainhead of future analyticity and science in Europe, this rationale was distinguished by philosophy into two domains: "telos" or 
cosmic rationale as manifest in the knowledge of the external world, and "logos" or reflective reasoning, a mirror for this cosmic rationale in the process of coming to understand. Much of Western theorizing up until the post-Medieval eras seems to have presumed the rationalizability of existence, leaving very few if any records of investigating arational phenomena such as instinct, the unconscious, value enculturation, or the technicalities of empirical unpredictability. Natural philosophy addressed the rational principles of our planet and supposedly geocentric cosmos; human nature was queried in terms of logic, social history, also practical disciplines such as politics and ethics; and metaphysics held on to the dream of uniting all knowledge within the scope of essential principles.

During the European Renaissance, technical thinking which had been exercised in quantification and engineering for millennia began to blend with efforts to understand the "natural laws" of the cosmos, motivating the invention of better observational tools such as telescopes. By the Scientific Revolution of the 16th and 17th centuries, telescope technology had become potent enough to revolutionize the European model of reality, as it was proven by direct visual evidence that humans do not live in a small pocket of the empyrium tailored for terrestrial life, but rather a vast spatial universe in relationship to which the race is an almost inconsequential speck, despite our privileged place in the dominance hierarchy of Earth's organisms.

Many of the first mathematical models were addressed to simple, intuitive phenomena such as the revolving of planets in our solar system, and fit the data with little equivocality, but as groundbreaking protoscience proceeded, the error which could arise in association with quantitative description grew more salient. This attention to recurring uncertainty readily conjoined with the nominalist heritage of Middle Ages Ockhamism, together engendering a paradigm viewing mathematical representations and eventually empirical explanation in total as an approximation, intrinsically subject to imprecision. Early Modern philosophers of the 17th century reflected upon the phenomenon of fallacious observation with some care, a skepticism that questioned many essentialist precedents and commenced the reinterpretation of empirical discovery as more a matter of methodology than revealed truth, a function of humanity's reasoning capabilities instead of metaphysical rationale.

The impossibility of using direct measurement to analyze most geometric figures for purposes of quantitative modeling was a barrier, but Newton (b. 1643) brought resolution with the invention of calculus, a method enabling empiricists to make infinitely precise approximations without measuring, solely by the manipulation of symbolic expressions. This opened up an unbounded world of mathematical form to modeling efforts, setting the stage for modernity's physics, astronomy, chemistry, geology and biology.

Leibniz (b. 1646), also a genius of deduction who independently invented calculus without however devoting it to empirical objectives, likewise realized the relevance of symbolic systems for constructing representations of our world, as 
well as in analyzing the logic of language. His very early experimentation with new metalanguages for truth-value, inspired by a long tradition of mathematical proof, foreshadowed the late-19th century innovations of Peirce (b. 1839) and Frege (b. 1848), their robust systems of symbolic logic expanded by analytic philosophy, particularly philosophy of language and philosophy of mind. Technical idioms platformed the development of logical architectures that when wedded to number theory and integrated into technological designs seeded electronics, computers, and a great leap forward to the Information Age.

In the 18th century, analysis of reason, the organs of human knowing, made great strides, inspired by proto-scientific shifts in worldview that cast doubt on the possibility of an observer-neutral explanatory framework in the mold of essentialist tradition. Kant (b. 1724) crowned the Early Modern discoursing that had fleshed out this philosophical skepticism with his Idealism, drawing up an epistemological theory which gave the first comprehensive treatment of how the world interrelates with human minds as we acquire knowledge. This seminal schema reinterpreted truth as begotten by categories of reason, the structure of conceptualization, prefiguring psychology and neuroscience. Its acknowledgement of the limitations that cognition places on our ability to define reality in terms of absolute structure dealt the decisive blow to system-building metaphysics, so that future philosophies would be much more intuitionist, obligated to qualify insights about nature and the universe with reference to relativities of perceptual and cultural perspective, the discrepancies between apparent and actual causality as well as amongst individuals. In the 19th and 20th centuries, Kant's anatomy of reason would be enriched by the new fields of phenomenology and its offshoot mereology in a similar delineation of the conceptual categories associated with logic, identity and more.

Also around this 18th century period, European population was expanding fast, with subcultural diversity a major policy issue, as even minority demographics had grown to hundreds of thousands if not millions strong. It was necessary to assess the new dynamics of these larger societies, finding ways to mobilize the masses, prevent unrest, and predict long-term ramifications. Philosophers studied human nature, economy, political systems and ethics as reconstituted by sprawling multitudes, with mainstream thought evolving towards more egalitarian ideologies committed to offering concessions and broadened participation, appeasing the irrepressible public with opportunities to advocate for its own interests through legal channels and reap maximum benefit from institutions, ultimately for the goal of sustaining countries' intellectual and technical formidability in the rivalries of Europe.

As knowledge spread under the influence of egalitarianism and the continent started to academicize, analysis of literature increased in sophistication. Philosophy was growing more conscious of historical context as evinced by written records, a cognizance of the way culture differs by time, place, the idiosyncrasies of various individuals and collectives. At first this burgeoning relativism by and 
large refused to relinquish the age-old ultra-rationalist bent of high-level thinking, resulting in preponderant Hegelianism, a paradigm regarding historical development as the interaction of sequentially parallel forms that self-organize in relatively neat and tidy, "thesis", "antithesis", "synthesis" arrangements, as if analogous to dialectical reasoning. Marx (b. 1818) customized this notion to develop Communist theory, integrating dialecticism with the somewhat messy facts of European economic history to propose a hypothesis about the path social development was likely to take in the future. Dialecticism must have been diffusing into the intellectual milieu at large, for a field biologist named Darwin (b. 1809) applied the same idea to naturalism, a completely distinct academic area, setting forth a model of how taxonomy changes by way of selection pressures that interact as they propagate in parallel, and this theory of evolution provided the foundation for life science of the future-molecular genetics, environmental ecology, evolutionary psychology-while uniting all of modern biology to date within a single paradigm.

In the 19th century, European philosophers dedicated their energy to analyzing the arationality of existence, a theme which had mostly been ignored until exploding population and the complicacy of imperial rule brought arbitrariness in human life and practice to the foreground. A movement that purveyed philosophy as literature gained a following, championed by Nietzsche (b. 1844) and others, which refrained from filtering the relativistic aesthetics of modern life out of philosophical reasoning in order to more organically, holistically portray the nature of truth while refusing to expunge subjectivity of the willing agent from the inquiry. This "Romanticism" strongly influenced the intellectual climate, with its surreal ultra-realism stimulating more conventionally analytical fields such as medicine and field biology to show interest in phenomena that had previously been shunned, such as madness, irrationality and the organic causes of immorality, touching off a theoretical parsing of the psyche into arational functions by new research disciplines like linguistics, psychology, sociology, anthropology and cognitive science. Its literary dimension continued on despite trajection in the 20th century towards a society centered on high technology and technical professionalism, spawning academic movements of structuralism, modernism and postmodernism which worked to deconstruct the evolution of motive, knowledge, belief and valuation as exhibited in records of the memetic past.

So if there is an overall direction within the multimillennial progression of our episteme, it seems this can be summed up as a move from essentializing, reductionistic pursuit of absolute truth to more pragmatic and adaptive methodologies in all kinds of untapped areas. Our conceptualizing of the world has become more conscious of perspective, the dependence of knowledge and appearance on both a metamorphosizing nature of the observer as well as the context within which reality is viewed. Thanks to academia, humans suffer much less from the impression that language, due to rhetorical skill, abstruseness or unfamiliarity, is 
indisputable, coercive or insurrectional rather than consisting in tentative proposals to be mutually interrogated. The public more collaboratively and creatively shares in building theory instead of submitting to doctrine, ignoring ingenious ideas, or resisting epistemic change.

At this point, we can muster a developmental phenomenology of truth in expression, tracing the cultural evolution of explicit conceptualizing within European civilization. During prehistory, humans spent much of their time solving technical problems that environments and societies posed, fixating on the constituent elements of assorted contexts when some sort of perplexity became apparent, then mastering those local conditions with the application of creative reasoning. Our species also projected affective states and sensations, intellectually refined in comparison to most of Earth's organisms, into social relationships and reality in general as personal and cultural valuation. Narrative was one of the primary vehicles for expressing values, an aesthetic of artistry and verity that organized symbolic utterance as a cultural motif, giving voice to some of the most inspired and high functioning mental states. Thousands of years were required before these two avenues for expressing the human spirit would merge, yet by the latter half of the first millennium B.C.E., technicalized thinking and storytelling traditions had melded into an analytical discourse which did not merely assign value to experiences, but sought to subsume them with an exhaustive explanation. Expression was no longer impetuous though cathartically actualizing will engaging with the world, but an incisive conquest of nature, culture and one's own intellectual frailties, which tried to unravel the Gordian knot of existence by delving into its essence with reasoning, upholding an ideal of supreme, incontestable accurateness as mastery of one's psychological milieu. Self and communal fulfillments effectuated by the pursuit of objective truth motivated steady philosophical progress until ancient Greeks and others had given accountings of reality's structure and principles that still hold some elucidative weight in our 21st century.

The model of a static cosmos promulged by Greeks such as Aristotle held an unquestioned place in human understanding until 14th century Medieval Ockhamism, when a contrasting idea was entertained that natural philosophy and associated expository expressing are more approximative and less a reflective mirror of the supposedly static cosmos. Dismantling geocentrism during the Renaissance and replacing it with the heliocentric model proved that the mind's image of reality can change in major ways, with technical approximations being not only partial but liable to systemic error. Due to this insight, a philosophy of skepticism came to prominence that questions and revises models, even sometimes going so far as to doubt the very possibility of certainty. Systematic methodologies such as those of calculus and eventually statistics provided a way to rank models according to plausibility (accuracy) and approximational soundness (precision), which made reasoning the obvious locus of knowledge and put external essence in a subsidiary position. This culminated in philosophies that 
propounded the mind's interpreting of nature as the decisive factor in epistemic structure.

As theories of dialectical history and especially physical evolution entered the picture, it became demonstrable that the fleeting certainty of modeling is not an anomaly, but mutation and metamorphosis are intrinsic to the total structure of reality. All models have thus far been temporary in their validity due to the conditions of both our world and ourselves, so that we must be willing at all times to amend these models in order to move towards better compensations for systemic fallacy as epistemic contexts falsify.

The modern consequence of knowledge's tentativeness was that language which expresses it began to seem provisional as well. Academic discourse does not consist in fundamental, unerring truths to be proclaimed as an absolute authority, but even the most meticulous verbalizations, in scholarly writing and elsewhere, have a degree of imprecision like the approximational modeling which they describe. Even the strongest rhetoric is hypothetical, and acceptance of this truism is an epiphany that breaks down submissiveness to orthodoxy, allowing humans to fully embrace unincarnated possibility, epistemic progress and paradigmal obsolescence with alacrity, gaining a concept of self-determination that maximizes the willpower necessary to strategize a remodeling of our world. This is the essence of enlightenment's intellectual dimension: the symbols we use to conceive existence and communicate our understandings to others are not representations of the indubitable, but rather a tool we wield which can be harnessed to serve actualization of the self. Grasping the idea of expressed knowledge's conditionality and arbitrariness equips the psyche to see how it can shape this arbitrarity into a boundless variety of forms to suit its interests.

Academic logic's history provides a perfect example of the movement from the stasis of long precedential essentialisms to adapting theoretical positivism. Aristotle's 4th century B.C.E. syllogistic logic was an essentialist model of persuasion, reducing natural language to meta-statements, grammatically abbreviated "premises" as for instance "Socrates is a man" and "all men are mortal", containing atomic factual "terms" such as "Socrates", "man" and "mortal", from which were derived "conclusions" like "Socrates is mortal", altogether including a narrow collection of inference types generalizing how a large range of explicit assertions are true or false (Editors of Encyclopedia Britannica, n.d.). No indication is given that it should be regarded as a conditional, temporary approximation; the model is proposed with faith in its legitimacy, as a function of broad scope and self-consistency, for Aristotle even goes so far as to declare non-contradiction the primary criterion of well-formed deduction. At this stage of ancient Greek academia, critique of rhetoric was committed to the tenet that efficacious theories imply an absolute ontology.

Chrysippus (b. 276 B.C.E.) and his fellow Stoics invented propositional logic, more closely examining the properties of true and false statements. They began to refine the Aristotelian term framework into an algebra-like format, inspired 
by advancements in mathematical proof, translating assertions of atomic fact along with their connective inferencing into metasymbolic denotation, much later reworked into a comprehensive technical system, e.g. $\mathrm{S} \wedge \mathrm{P}$ (either $\mathrm{S}$ or $\mathrm{P}$ ), S \& P (both S and P), etc., (Internet Encyclopedia of Philosophy, n.d.). This transitioned logic from Aristotle's technical rhetoric of apt deduction's supposed essence to logic as a progressional methodology, still looking to define some kind of essence, but with ascertainment that the modeling context is not absolute fidelity, instead modifiable as a series of improvements. This was an early infusion of Kantianlike thinking, the critical and revisionary perspective on system-building, into a branch of philosophy, as well as insertion of certain Platonic-styled concepts of pure form, such as the aforementioned bifurcation (disjunction) and recombination (conjunction), into the formulation of inference rules. It hybridized philosophical intuitions about the nature of structural form, which had been enhanced by materialism, with language-like truth-value.

After some sporadic, nonsymbolic dabblings in the field as well as a barren period from the 15th to the 19th century, Frege worked out a complete system of predicate logic at the turn of the 20th, with a fully algebraic notation similar to that used for formal deduction and proof in mathematics. Its grammar was entirely contrived, with no likeness at all to natural language; to the extent that logicians employed it in representing linguistic expressions, this was carried out by translation into a technical symbolism which is undecipherable to even a fluent speaker unless instructed. Predicate logic eliminates all phonetic properties of natural language that are superfluous to concrete meaning, such as prepositions, conjunctions, tense or gender, arranging nouns and verbs in maximally compact formulas, e.g. "Sxy", where " $S$ " stands for the predicate of a sentence, while the variables " $x$ " and " $y$ " symbolize subject and object, with the meaning of the formula determined by arbitrary rules of inference for variable relationships, explicitly specified at the outset. These "well-formed formulas" are mixed and matched into compound statements expressing complex inferential relationships, and then tandems of statements can be manipulated through a series of steps to perform deductive proofs in an economical manner, exactly as in modern math (Nolt, 2012). Philosophically speaking, predicate logic reduces expressions to the objects they are about, interpreted as subsisting within the boundaries of a contextual universe-“humans", "animals", "quantities"-with its specific criteria for inferential possibility. It is a concise, multipurpose way of describing objective truth-value and modeling relations between sets of existents.

This system was the next step in liberation from essence-conceptualizing, not merely malleable enough to encompass an ample breadth of meaningful forms like propositional logic, but at least in principle capable of being adapted to fit the object-context of any common, technical or symbolic language, old or new, with clear cognizance that it is a designed, pragmatic instrument, a so to speak technology of representation, not a naturalistic or exceptionally ontological entity. Yet predicate logic was still spellbound by a measure of essentialist com- 
mitment, namely the constrainment of object-contexts as such to the spatiotemporal frame of reference, attaching it to some naive realism ground rules for the nature of factual content, existents distinguished, acting and reacting while occupying mutually exclusive positions (law of the excluded middle) and in noncontradictory ways (law of noncontradiction), an interpretation of variables as consisting in forms analogous to material objects with their definite shapes, sizes and locations, basically a grammatical template for inferencing about an essentially corporeal rather than theoretical reality. It reified spatiotemporality, the conceptual substrate of mechanism as well as inorganic and organic matter in the sense-perceptual world, which is intrinsic to technological thinking and body awareness insofar as they are linked with properties of aggregate mass in Earth environments, erecting a technical idiom upon the functionally powerful but philosophically flawed premise that our macroscopic behavioral medium is of fundamentality in the phenomenal world.

The notion that mechanistically material, "physical" causality is foundational essence gives us a practical reductionistic chassis for many theoretical and engineering purposes, but when we extol essentialist reductionism of this or any type as philosophical doctrines, even with the recognition that essence evolves, it can hamper imagination in the inexperienced thinker. While physicalist essentialism was perhaps viable for making academic knowledge more intuitive in an early 20th century epoch of primordial science and undereducated populaces, it has become a stumbling block to intellectual progressiveness that the culture of objectivity probably needs to overcome. Physicalism's drawbacks can be remedied by academicizing which presents modern knowledge in a more balanced, multifaceted way, contextualizing physicalism along with all paradigmal camps such that we stimulate mind-expanding perspectivizing instead of ossificational essentializing.

Revival of formalism at the turn of the 20th century was in part a critical response to the 19th century psychologism that believed an acceptable theory of logic would be based on knowledge of the psyche. Logicians such as Frege and Edmund Husserl argued that this radically intuitionist paradigm, by viewing logic as psychical rather than a universalizable mode of reasoning, had subjected it to all kinds of unscientific and pernicious presumptions about the human spirit, heredity, instinct, predisposition, discrepancies between subcultures, ethnicities and individuals, an obsession with thinking of rationality as determined by preternatural or sociobiological aptitude, fomenting a nativist relativism anathema to objectivity. Proponents of the analytical paradigm as well as those of Kantian-inspired, phenomenological Idealism regarded psychologism as a debasement of academia with the nihilism that some such as Nietzsche had warned might one day engulf Europe.

Rapid improvements in the discipline of formal logic spurred its exponents towards the ambitious goal of forging a comprehensive theory of truth-value. Russell (b. 1872) and Whitehead (b. 1861) coauthored Principia Mathematica, a three-volume work which assigned itself the task of uniting all extant mathemat- 
ics within a single theoretical system of meta-principles and formal symbolic notation under the assumption that methodologies and techniques of quantification are based on a self-coherent universe of logical inferences and operations (Stanford Encyclopedia of Philosophy, 2021). Then in the 1930s a movement called logical positivism came together in Europe, influenced by philosophers of the Vienna Circle, taking on the objective of grounding mathematics and scientific research on logical foundations, with a symbolic language suitable to all theoretical efforts, a universal framework for objectivity (Encyclopedia.com., 2018). While this paradigm invigorated research in the field of logic, it succumbed to the quagmire of essentialism, asserting that the only meaningful questions in philosophy are those formulatible in its terminological system, which if embraced as standard methodology would have expunged subjectivity, literature, and much of historical metaphysics from the mainstream inquiry. The consequences did not turn out to be this immoderate, but nevertheless induced a divergence of "Analytic" philosophy with its anchorage in formal logic from the rest of the philosophical tradition, along with a possible role in inciting the sharp divide between hard sciences and the humanities that persists to this day.

Many of these analytic logicians, despite throwing a lasting wedge between their own work and that of other sub-disciplines, ended up eating their words, moving over some decades towards an approach which assessed truth-value with greater naturalism, considering the way logical meaning is bound to and varies by expressive context, in line with discrepancies of function and epistemic history, until all delimitations of actual and potential possibility in the field nearly evaporated. Academic logic had proceeded through multiple stages: Aristotle's ontological model of pure deduction's essence, the Stoics' progressive, more technicalized model of truth-value's essence as it subsists organically in expression, Frege's system for flexibly modeling supposed spatiotemporal essence, the logical positivist prospection into a system of discourse rendering transparent the essence of all theoretical structure, and finally bursting out of essentialist chains entirely in a discourse that is perspectivist to the core, with its unprecedented and growing versatility.

Research in the discipline of logic expanded into numerous specializations, particularly many that are affiliated with math. Intuitionist logic eliminated the law of the excluded middle from its inferential systems, which was useful for constructing mathematical proofs with nonspatial entities (Stanford Encyclopedia of Philosophy, 2018a), and paraconsistent logics provided inferential systems which even omitted the law of noncontradiction, applied in modeling the structure of conflicting arguments among much else (Stanford Encyclopedia of Philosophy, 2018b). Around this same post-WW2 era, the rest of the Western intelligentsia was also diversifying into new areas, with every academic department dividing into dozens if not hundreds of subfields. Students obtained the license to design their own specialty by immixing degree programs, accommodating a wide range of interests and points of view. 
Much of modernity was truly beginning to get it: epistemic development loosened itself from the fetters of an essentializing that places greater value on doctrinal solidarity than practicality or progress, as well as from the rhetoricality which only accentuates this habitude and even deludes many of the academics who articulate scholarly rhetoric into dogmatic stagnation. With public schooling, pedagogical literature, and online resources that put most of the world's knowledge within reach in a matter of seconds, intellectual enlightenment is accessible to nearly everyone. Humanity at least in principle has access to the information that will empower mastery of any academic subject and fulfill our potential. Even individuals of average means commonly grasp how they can make themselves into cognoscente via diligent study, and our cultures are so saturated with technical facts and theory that expertise in at least some areas is virtually unavoidable. Human willpower is far more cerebral than it ever has been, with polymathic enlightenment costing a small fraction of one's paycheck in the U.S. Contemporary knowledge has brought about massive increases in the intellectual agency for our cultures, and the 21st century will be tasked with integrating these competencies into the institutional structure so as to actualize the species, blazing a trail to an age of high technology and human legacy.

\section{Conflicts of Interest}

The author declares no conflicts of interest regarding the publication of this paper.

\section{References}

April, H. (2013) Ancient Origins. Neanderthals Buried Their Dead in Ancient Ritual. https://www.ancient-origins.net/news-history-archaeology/neanderthals-buried-theirdead-ancient-ritual-001130

Australian Antarctic Program (n.d.). Emperor Penguin Breeding Cycle. https://www.antarctica.gov.au/about-antarctica/animals/penguins/emperor-penguins/b reeding-cycle/

Berkeley.edu (n.d.). Understanding Evolution. https://evolution.berkeley.edu/evolibrary/article/adaptiveradiation_01

Berwick, R. C., Okanoya, K., Beckers, G. J. L., \& Bolhuis, J. J. (2011). Songs to Syntax: The Linguistics of Birdsong (Review). Trends in Cognitive Sciences, 15, 113-121.

https://www.sciencedirect.com/science/article/abs/pii/S1364661311000039

Bond, E. (2020a). The Conjoined Evolution of Conception, Language and Sociality. https://philosophyofhumanism.com/2020/12/04/the-conjoined-evolution-of-conceptio n-language-and-sociality/

Bond, E. (2020b). The Nature and Human Impact of Qualia. http://philosophyofhumanism.com/2020/11/16/the-nature-and-human-impact-of-qualia/

Bond, E. (2020c). The Origins and Evolution of Perception in Organic Matter. http://philosophyofhumanism.com/2020/11/17/the-origins-and-evolution-of-perceptio n-in-organic-matter/

Bosak, L., Moore, A., Masino, C., \& Klein, A. (2013). Foraging and Feeding Behavior of the Eastern Grey Squirrel (Sciurus carolinensis). Department of Biology, University of Maryland.

http://science.umd.edu/classroom/bsci338m/MdMammalogy/Bosak_et_al_2013.pdf 
Boyer, C. B. (1991). A History of Mathematics. John Wiley \& Sons, Inc.

Cloud, D. (2015). The Domestication of Language: Cultural Evolution and the Uniqueness of the Human Animal. Columbia University Press.

Editors of Encyclopedia Britannica (n.d.). Syllogistic Logic. https://www.britannica.com/topic/syllogistic

Editors of Encyclopedia Britannica (n.d.). Humpback Whale. https://www.britannica.com/animal/humpback-whale

Elephant Voices (n.d.). Acoustic Communication. https://www.elephantvoices.org/elephant-communication/acoustic-communication.html

Encyclopedia.com. (2018). Logical Positivism. https://www.encyclopedia.com/philosophy-and-religion/philosophy/philosophy-terms -and-concepts/logical-positivism

Erard, M. (2018). Is This 100,000 Year Old Hashtag the First Humanmade Symbol or Just a Pretty Decoration? https://www.sciencemag.org/news/2018/04/100000-year-old-hashtag-first-human-sym bol-or-just-pretty-decoration

Extinct Animals (n.d.). Brachiosaurus. https://www.extinctanimals.org/brachiosaurus.htm

Hampton, D. (2019). 6 Basic Principles of Neuroplasticity. https://thebestbrainpossible.com/6-basic-principles-of-neuroplasticity/

Harcourt, A. H. (2015). Humankind: How Biology and Geography Shape Human Diversity. Pegasus Books.

History.com. (n.d). Neolithic Revolution. https://www.history.com/topics/pre-history/neolithic-revolution

Internet Encyclopedia of Philosophy (n.d.). Propositional Logic. https://iep.utm.edu/prop-log/

Kandalintseva, D. (2012). Animal Communication Lions. https://www.slideshare.net/DariaKandalintseva/animal-communication-lions

Linguistic Society of America (n.d.). How Did Language Begin? https://www.linguisticsociety.org/resource/faq-how-did-language-begin

Lumen: Boundless Psychology (n.d.). Introduction to Human Language. https://courses.lumenlearning.com/boundless-psychology/chapter/human-language/

Mann, C. C. (2005). 1491. Random House Inc.

Mark, J. J. (2009). World History Encyclopedia: Literature. https://www.worldhistory.org/literature/

Mayntz, M. (2019). Bird Mimics and Mimicry. https://www.thespruce.com/bird-mimics-and-mimicry-386219

McFadden, J. (2021). Does Consciousness Come from the Brain's Electromagnetic Field? https://aeon.co/essays/does-consciousness-come-from-the-brains-electromagnetic-field

My Animals (n.d.). Do Parrots Understand What They Say? https://myanimals.com/animals/domestic-animals-animals/birds-and-lovebirds/do-par rots-understand-what-they-say/

Nolt, J. (2012). Logic: Schaum's Easy Outlines. McGraw-Hill.

PBS (n.d.). The Hominid Family Tree. http://www.pbs.org/wgbh/evolution/humans/humankind/j.html

Pearsonhighered.com. (n.d.). The Beginnings of Civilization. https://www.pearsonhighered.com/assets/samplechapter/0/1/3/2/0132132850.pdf 
Pobiner, B. (2016). The First Butchers.

https://www.sapiens.org/biology/homo-sapiens-and-tool-making/

Rice, S. A. (2011). Life of Earth: Portrait of a Beautiful, Middle-Aged, Stressed-Out World. Prometheus Books.

Rice.edu. (n.d.). The Origin and Evolution of Human Language: Homo Habilis and Homo Erectus. http://www.ruf.rice.edu/ kemmer/Evol/habiliserectus.html

Schmandt-Besserat, D. (2014). The Evolution of Writing. In Wright, James (Ed.), International Encyclopedia of Social and Behavioral Sciences (pp. 1-15). Elsevier.

https://sites.utexas.edu/dsb/tokens/the-evolution-of-writing/

Science.smith.edu. (n.d.). Migration of Humans into the Americas. http://www.science.smith.edu/climatelit/migration-of-humans-into-north-america/

Scientific Psychic (n.d.). Geologic and Biological Timeline of the Earth. https://www.scientificpsychic.com/etc/timeline/timeline.html

Smith, D. (2002). The Evolution of the Unconscious. Psychoanalytische Perspectieven, 20, 533-536.

https://www.psychoanalytischeperspectieven.be/vol-20-d-2002/the-evolution-of-the-un conscious

Stanford Encyclopedia of Philosophy (2018a). Intuitionistic Logic. https://plato.stanford.edu/entries/logic-intuitionistic/

Stanford Encyclopedia of Philosophy (2018b). Paraconsistent Logic. https://plato.stanford.edu/entries/logic-paraconsistent/

Stanford Encyclopedia of Philosophy (2021). Principia Mathematica. https://plato.stanford.edu/entries/principia-mathematica/

The National Wildlife Federation (n.d.a). Grizzly Bear. https://www.nwf.org/Educational-Resources/Wildlife-Guide/Mammals/Grizzly-Bear

The National Wildlife Federation (n.d.b). Monarch Butterfly. https://www.nwf.org/Educational-Resources/Wildlife-Guide/Invertebrates/Monarch-B utterfly

Torrey, F. E. (2017). Evolving Brains, Emerging Gods, Early Humans and the Origins of Religion. Columbia University Press.

Tropical Essays (2011). The Emergence of Bipedal Hominids. https://tropicalessays.com/emergence-bipedal-hominids/

University of Gothenburg (2020). Human-Caused Biodiversity Decline Started Millions of Years Ago.

https://phys.org/news/2020-01-human-caused-biodiversity-decline-millions-years.html

Welker, B. H. (n.d.). The History of Our Tribe: Hominini. Creative Commons Attribution NonCommercial ShareAlike.

https://milnepublishing.geneseo.edu/the-history-of-our-tribe-hominini/

Whipps, H. (2008). How Writing Changed the World.

https://www.livescience.com/2283-writing-changed-world.html

Whitaker, R. (2018). Did You Know that Dolphins Have Names?

https://animalogic.ca/blog/did-you-know-that-dolphins-have-names

Williams, H. (n.d.). Bird Song and Singing Behavior. Department of Biology. Williams College. https://biology.williams.edu/files/2013/05/HWchapter.pdf

Worldlife (n.d.). Sloth. https://www.worldwildlife.org/species/sloth 